\title{
Joint Wall Mitigation and Compressive Sensing for Indoor Image Reconstruction
}

\author{
Eva Lagunas, Student Member, IEEE, Moeness G. Amin, Fellow, IEEE, \\ Fauzia Ahmad, Senior Member, IEEE, and Montserrat Nájar
}

\begin{abstract}
Compressive sensing (CS) for urban operations and through-the-wall radar imaging has been shown to be successful in fast data acquisition and moving target localizations. The research in this area thus far has assumed effective removal of wall electromagnetic backscatterings prior to CS application. Wall clutter mitigation can be achieved using full data volume which is, however, in contradiction with the underlying premise of CS. In this paper, we enable joint wall clutter mitigation and CS application using a reduced set of spatial-frequency observations in stepped frequency radar platforms. Specifically, we demonstrate that wall mitigation techniques, such as spatial filtering and subspace projection, can proceed using fewer measurements. We consider both cases of having the same reduced set of frequencies at each of the available antenna locations and also when different frequency measurements are employed at different antenna locations. The latter casts a more challenging problem, as it is not amenable to wall removal using direct implementation of filtering or projection techniques. In this case, we apply CS at each antenna individually to recover the corresponding range profile and estimate the scene response at all frequencies. In applying CS, we use prior knowledge of the wall standoff distance to speed up the convergence of the orthogonal matching pursuit for sparse data reconstruction. Real data are used for validation of the proposed approach.
\end{abstract}

Index Terms-Compressive sensing (CS), through-the-wall radar imaging, wall mitigation.

\section{INTRODUCTION}

$\mathbf{T}$ HROUGH-the-wall radar imaging (TWRI) is an emerging technology of rising interest with the objectives of sensing through the wall and inside enclosed building structures using electromagnetic (EM) waves. The sensing could determine the building layouts, discern the intent of activities inside the building, and detect, identify, and track moving targets [1]-[19]. In order to detect and localize targets, the clutter, caused by

Manuscript received February 16, 2012; revised April 19, 2012; accepted June 3, 2012. Date of publication July 23, 2012; date of current version January 17, 2013. The work by M. G. Amin and F. Ahmad was supported in part by the Office of Naval Research under Grant N00014-11-1-0576 and in part by the Army Research Office and Army Research Laboratory under contract W911NF-11-1-0536. The work by E. Lagunas was performed during her tenure as a Visiting Research Associate at the Center for Advanced Communications, Villanova University. E. Lagunas is partially supported by the fellowship FIDGR-2011. M. Nájar is partially supported by the Spanish Ministry of Science and Innovation (Ministerio de Ciencia e Innovación) under project TEC201129006-C03-02 (GRE3N-LINK-MAC).

E. Lagunas and M. Nájar are with the Signal Theory and Communications Department, Universitat Politècnica de Catalunya, 08034 Barcelona, Spain (e-mail: eva.lagunas@upc.edu; montse.najar@upc.edu).

M. G. Amin and F. Ahmad are with the Radar Imaging Lab, Center for Advanced Communications, Villanova University, Villanova, PA 19085 USA (e-mail: moeness.amin@ villanova.edu; fauzia.ahmad@villanova.edu).

Color versions of one or more of the figures in this paper are available online at http://ieeexplore.ieee.org.

Digital Object Identifier 10.1109/TGRS.2012.2203824 the front wall EM backscatterings, must be suppressed, or significantly mitigated, and its effects on the imaged scene accuracy and fidelity must be limited.

Behind-the-wall target returns are relatively weak compared to front wall reflections and, therefore, stationary targets cannot be generally detected without an effective removal of front wall clutter. Attempting to remove the front wall reflections from the received data by change detection [20]-[22] cannot be performed for stationary scenes. This is because the subtraction of consecutive imaging results would eliminate both target and clutter.

In TWRI, backprojection is typically employed for image formation. Recently, it has been shown that compressive sensing (CS) and $l_{1}$ norm reconstruction techniques can be applied, in lieu of backprojection, to reveal the target positions behind walls. In so doing, significant savings in acquisition time can be achieved. Further, producing an image of the indoor scene using few observations can be logistically important, as some of the data measurements in space and frequency can be difficult, or impossible to attain. In this paper, we apply joint CS and wall mitigation techniques using reduced data measurements. In essence, we address wall clutter mitigations in the context of CS.

The application of CS for TWRI was first reported in [23] and further developed in [24]-[26]. However, wall mitigation in conjunction with CS has never been considered in these references. That is, CS was applied to TWRI, already assuming prior and complete removal of the front wall EM returns. Without this assumption, strong wall clutter, which extends along the range dimension, reduces the sparsity of the scene and, as such, impedes the application of CS. If the reference scene is known, then background subtraction can be performed to remove wall clutter, thereby improve sparsity. Having access to the background scene or reference data, however, is not always possible in practical applications.

There are several approaches which successfully mitigate the front wall contribution to the received signal. These approaches were originally introduced to work on the full data volume and did not account for nor were they ever examined under reduced data measurements. They can be categorized into those based on estimating the wall parameters and others incorporating either wall backscattering strength or invariance with antenna location [9], [27]-[30]. In [9] and [27], a method to extract the dielectric constant and thickness of the nonfrequency-dependent wall from the time-domain scattered field was presented. The time-domain response of the wall was then analytically modeled and removed from the data. In [28], a 
spatial filtering method was applied to remove the dc component corresponding to the constant-type radar return, typically associated with the front wall. The third method, presented in [29] and [30], was based not only on the wall scattering invariance along the antenna locations but also on the fact that wall reflections are relatively stronger than target reflections. As a result, the wall subspace is usually captured in the most dominant singular values when applying singular value decomposition (SVD) to the measured data matrix. The wall contribution can then be removed by orthogonal subspace projection.

In this paper, we examine the performance of the spatial filtering and subspace projection wall mitigation techniques in conjunction with sparse image reconstruction. Only a small subset of measurements is employed for both wall clutter reduction and image formation. The wall parameter estimation and backscattering modeling technique is outside the scope of this paper. Unlike the two other techniques, strong antenna ringing hinders EM modeling and prevents performance validation experimentally in our lab.

We consider two cases of frequency measurement distributions over antenna positions. In the first case, the same subset of frequencies is used for each antenna. The other case allows the frequencies to differ from one antenna to another. For the subspace projection and spatial filtering methods, we show that when the same subset of frequency measurements is used at each antenna, those two methods maintain their proper performance as their full-data set counterparts. CS techniques for image reconstruction can then be applied with the same reduced measurements but of much higher signal-to-clutter ratio. On the other hand, using different frequencies at different antenna positions would impede the application of either method. This is because the phase returns across the antenna elements would be different, which deprives the wall mitigation algorithms of the underlying assumption of spatial invariance of the wall clutter. This problem is overcome in this paper by first reconstructing the range profile, which is based on $l_{1}$ norm minimization. This is performed at each antenna individually. Then, the data of the missing frequencies can be obtained by taking the FFT of the reconstructed range profile at each antenna. A similar approach was adopted in [25]; however, it required the fundamental assumption of prior removal of wall EM scattering. Once the phase returns corresponding to all original frequencies are estimated, wall mitigation can proceed using spatial filtering, subspace projection, or any other conventional wall mitigation method.

In this paper, sparse data reconstruction is performed using orthogonal matching pursuit (OMP) which provides fast $l_{1}$ solutions and is appropriate for stepped frequency radar imaging. Since the target is behind the wall, the OMP can be modified such that the iterations corresponding to the range up to the wall can be combined. This allows a quicker inclusion of the target into the reconstruction algorithm. We compare OMP with the modified OMP and show the abilities as well as the challenges of performing TWRI with arbitrary data measurements.

The remainder of this paper is organized as follows. Section II reviews CS fundamental formulation. Section III presents the through-the-wall signal model and provides the fundamental equations of the sparsity-based scene reconstruction. Section IV reviews the spatial filtering and subspace projection-based wall clutter mitigation techniques presented in [28] and [30], respectively. The joint wall mitigation and CS for TWRI is discussed in Section V. Finally, Section VI presents the performance results of the spatial filtering and the subspace projection in conjunction with CS and sparse image reconstruction using real-data collected in a semi-controlled environment. Conclusions are drawn in Section VII.

\section{COMPRESSIVE SENSING}

CS theory states that a sparsely representable signal can be reconstructed using fewer measurements compared to the signal dimension [31]-[33]. For a brief review of the main ideas underlying CS, consider a finite length, discrete time signal $\mathbf{x} \in \mathbb{C}^{N}$, which can be expressed in an orthonormal basis $\Psi=\left[\begin{array}{llll}\boldsymbol{\psi}_{1} & \boldsymbol{\psi}_{2} & \ldots & \boldsymbol{\psi}_{N}\end{array}\right]$ as follows:

$$
\mathbf{x}=\sum_{i=1}^{N} \boldsymbol{\psi}_{i} \theta_{i}
$$

In (1), the vector $\boldsymbol{\theta}=\left[\begin{array}{llll}\theta_{1} & \theta_{2} & \ldots & \theta_{N}\end{array}\right]^{T}$ is a sparse vector, which means that it only has $K(K \ll N)$ non-zero components. Using matrix notation, we can express $\mathrm{x}$ as

$$
\mathrm{x}=\boldsymbol{\Psi} \theta .
$$

Consider only $Q=O(K \log (N / K))$ linear non-adaptive samples of the signal $\mathbf{x}$, i.e., $\breve{\mathbf{y}}=\boldsymbol{\Phi} \mathbf{x}$ where matrix $\boldsymbol{\Phi} \in \mathbb{C}^{Q \times N}$ with $K<Q \ll N$ is the sub-sampling matrix. The sparse signal $\boldsymbol{\theta}$ can be then recovered from $\breve{\mathbf{y}}$ by solving the following optimization problem:

$$
\min _{\boldsymbol{\theta}}\|\boldsymbol{\theta}\|_{l_{1}} \quad \text { subject to } \quad \breve{\mathbf{y}} \approx \boldsymbol{\Phi} \boldsymbol{\Psi} \boldsymbol{\theta}
$$

where $\|\boldsymbol{\theta}\|_{l_{1}}=\sum_{i}\left|\theta_{i}\right|$. Several methods are available in the literature to solve the optimization problem in (3). The $l_{1}$ minimization is a convex problem and can be recast as a linear program [34]. This is the foundation for the basis pursuit techniques [33], [35], [36]. Alternatively, greedy methods, known as matching pursuit (MP), can be used to solve (3) iteratively [37], [38].

\section{COMPRESSIVE SENSING FOR TWRI}

In this section, we first present the through-the-wall signal model, followed by a brief description of the sparsity-based scene reconstruction, highlighting the key equations.

\section{A. Through-the-Wall Signal Model}

Consider a homogeneous wall of thickness $d$ and dielectric constant $\epsilon$ located along the $x$-axis, and the region to be imaged located beyond the wall along the positive $z$-axis. Assume that an $N$-element line array of transceivers is located parallel to the wall at a standoff distance $z_{o f f}$, as shown in Fig. 1. Let the $n$th transceiver, located at $\mathbf{x}_{n}=\left(x_{n},-z_{o f f}\right)$, illuminate the scene with a stepped-frequency signal of $M$ frequencies, which are equispaced over the desired bandwidth $\omega_{M-1}-\omega_{0}$

$$
\omega_{m}=\omega_{0}+m \Delta_{\omega} \quad m=0, \ldots, M-1
$$




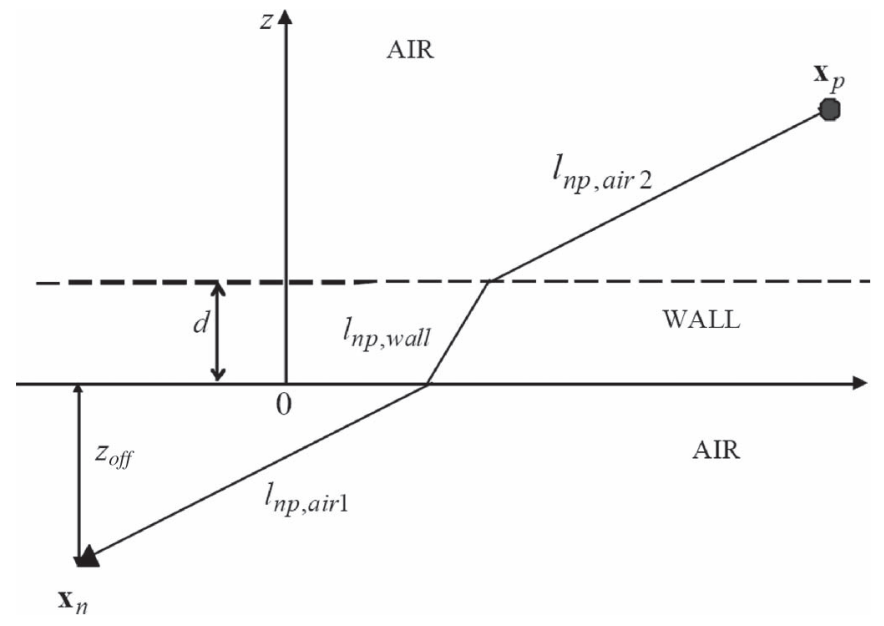

Fig. 1. Geometry on transmit of the equivalent 2-D problem.

where $\omega_{0}$ is the lowest frequency in the desired frequency band and $\Delta_{\omega}$ is the frequency step size. The reflections by the wall and any targets in the scene are measured only at the same transceiver location. Assuming the scene contains $P$ point targets, the output of the $n$th transceiver corresponding to the $m$ th frequency is given by

$$
y(m, n)=\sigma_{w} \exp \left(-j \omega_{m} \tau_{w}\right)+\sum_{p=0}^{P-1} \sigma_{p} \exp \left(-j \omega_{m} \tau_{p, n}\right)
$$

where $\sigma_{w}$ is the complex reflectivity of the wall, $\sigma_{p}$ is the complex reflectivity of the $p$ th target, $\tau_{w}$ is the two-way traveling time of the signal from the $n$th antenna to the wall, and $\tau_{p, n}$ is the two-way traveling time between the $n$th antenna and the target. It is noted that both the wall and target reflectivities are assumed to be independent of frequency and aspect angle. The propagation delay $\tau_{w}$ is given by

$$
\tau_{w}=\frac{2 z_{o f f}}{c}
$$

where $c$ is the speed of light in free-space. On the other hand, $\tau_{p, n}$ is given by [11]

$$
\tau_{p, n}=\frac{2 l_{n p, a i r 1}}{c}+\frac{2 l_{n p, w a l l}}{v}+\frac{2 l_{n p, a i r 2}}{c}
$$

where $v=c / \sqrt{\epsilon}$ is the speed through the wall, and the variables $l_{n p, a i r 1}, l_{n p, \text { wall }}$, and $l_{n p, a i r 2}$ represent the traveling distances of the signal before, through, and beyond the wall, respectively, from the $n$th transceiver to the $p$ th target.

An equivalent matrix-vector representation of the received signals in (5) can be obtained as follows. Assume that the region of interest is divided into a finite number of pixels $N_{x} \times N_{z}$ in crossrange and downrange, and the point targets occupy no more than $P\left(\ll N_{x} \times N_{z}\right)$ pixels. Let $r(k, l)$, $k=0, \ldots, N_{x}-1, l=0, \ldots, N_{z}-1$ be a weighted indicator function, which takes the value $\sigma_{p}$ if the $p$ th point target exists at the $(k, l)$ th pixel; otherwise, it is zero. With the values $r(k, l)$ lexicographically ordered into a column vector $\mathbf{r}$ of length
$N_{x} N_{z}$, the received signal corresponding to the $n$th antenna can be expressed in matrix-vector form as

$$
\mathbf{y}_{n}=\Psi_{n} \mathbf{r}
$$

where $\boldsymbol{\Psi}_{n}$ is a matrix of dimensions $M \times N_{x} N_{z}$, and its $m$ th row is given by

$$
\left[\boldsymbol{\Psi}_{n}\right]_{m}=\left[\begin{array}{ll}
e^{-j \omega_{m} \tau_{n, 00}} & \ldots e^{-j \omega_{m} \tau_{n, N_{x} N_{z}}}
\end{array} .\right.
$$

Considering the measurement vector corresponding to all $\mathrm{N}$ antennas, defined as

$$
\mathbf{y}=\left[\begin{array}{llll}
\mathbf{y}_{0}^{T} & \mathbf{y}_{1}^{T} & \ldots & \mathbf{y}_{N-1}^{T}
\end{array}\right]^{T}
$$

the relationship between $\mathbf{y}$ and $\mathbf{r}$ is given by

$$
\mathrm{y}=\Psi \mathrm{r}
$$

where

$$
\boldsymbol{\Psi}=\left[\begin{array}{llll}
\boldsymbol{\Psi}_{0}^{T} & \mathbf{\Psi}_{1}^{T} & \ldots & \mathbf{\Psi}_{N-1}^{T}
\end{array}\right]^{T} .
$$

\section{B. Sparsity-Based Data Acquisition and Scene Reconstruction}

The expression in (11) involves the full set of measurements made at the $N$ array locations using the $M$ frequencies. For a sparse scene, it is possible to recover $\mathbf{r}$ from a reduced set of measurements. Consider $\breve{\mathbf{y}}$, which is a vector of length $Q_{1} Q_{2}(\ll M N)$ consisting of elements chosen from $\mathbf{y}$ as follows:

$$
\breve{\mathrm{y}}=\Phi \mathbf{y}=\boldsymbol{\Phi} \Psi \mathrm{r}
$$

where $\boldsymbol{\Phi}$ is a $Q_{1} Q_{2} \times M N$ matrix of the form

$$
\boldsymbol{\Phi}=\operatorname{kron}\left(\boldsymbol{\vartheta}, \mathbf{I}_{Q_{1}}\right) \cdot \operatorname{diag}\left\{\varphi^{(0)}, \ldots, \varphi^{(N-1)}\right\} .
$$

In (14), "kron" denotes the Kronecker product, $\mathbf{I}_{Q_{1}}$ is a $Q_{1} \times$ $Q_{1}$ identity matrix, $\vartheta$ is a $Q_{2} \times N$ measurement matrix constructed by either uniformly or randomly selecting $Q_{2}$ rows of an $N \times N$ identity matrix, and $\varphi^{(n)}, n=0,1, \ldots, N-1$, is a $Q_{1} \times M$ measurement matrix constructed by uniformly or randomly selecting $Q_{1}$ rows of an $M \times M$ identity matrix. We note that $\vartheta$ determines the reduced antenna locations, whereas $\varphi^{(n)}$ determines the reduced set of frequencies corresponding to the $n$th antenna location. The number of measurements $Q_{1} Q_{2}$ required to achieve successful CS reconstruction highly depends on the coherence between $\boldsymbol{\Phi}$ and $\boldsymbol{\Psi}$. For the problem at hand, $\boldsymbol{\Phi}$ is the canonical basis and $\boldsymbol{\Psi}$ is similar to the Fourier basis, which have been shown to exhibit maximal incoherence [32]. Given $\breve{\mathbf{y}}$, we can recover $\mathbf{r}$ by solving the following equation:

$$
\hat{\mathbf{r}}=\arg \min _{\mathbf{r}}\|\mathbf{r}\|_{l_{1}} \quad \text { subject to } \quad \breve{\mathbf{y}} \approx \mathbf{\Phi} \boldsymbol{\Psi} \mathbf{r} .
$$

In this paper, we consider MP to solve (15). More specifically, we use the OMP, which is known to provide a fast and easy to implement solution. Moreover, OMP is better suited when frequency measurements are used [39]. OMP can be modified 
to take into account the specificities of through-the-wall radar problems. Prior knowledge of the wall standoff distance in the reconstruction algorithm can allow the OMP to reconstruct the target pixels in fewer iterations and thus speed up the convergence of the algorithm. A detailed description of the proposed modification of the OMP is given in the Appendix.

In this paper, we consider five different measurement strategies listed below. Reduced set of frequencies (antennas) means using a smaller number of frequencies (antennas) than those available.

(a) Measure all $M$ frequencies at each of the $N$ antenna locations.

(b) Measure the same reduced set of frequencies for all $N$ antenna locations.

(c) Measure the same reduced set of frequencies for a reduced set of antenna locations.

(d) Measure a different set of reduced frequencies for each of the $N$ antenna locations.

(e) Measure a different set of reduced frequencies for a reduced set of antenna locations.

Each of the above strategies has a corresponding $\boldsymbol{\Phi}$ matrix associated with it. When the full set of measurements is available [case (a)], $\boldsymbol{\Phi}$ equals an identity matrix of dimensions $M N \times$ $M N$. If the same reduced set of frequency measurements are used at each antenna location (case (b) and case (c)), then $\varphi^{(0)}=\varphi^{(1)}=\ldots=\varphi$ and $\boldsymbol{\Phi}$ takes the form

$$
\begin{array}{ll}
\boldsymbol{\Phi}=\operatorname{kron}\left(\mathbf{I}_{N}, \boldsymbol{\varphi}\right) & \operatorname{case}(\mathrm{b}) \\
\boldsymbol{\Phi}=\operatorname{kron}(\boldsymbol{\vartheta}, \boldsymbol{\varphi}) & \operatorname{case}(\mathrm{c})
\end{array}
$$

where $\mathbf{I}_{N}$ is an identity matrix of dimensions $N \times N$. For case (d), in which all antenna positions are used but different reduced frequency sets are emitted by different antennas, the expression in (14) reduces to

$$
\boldsymbol{\Phi}=\operatorname{diag}\left\{\varphi^{(0)}, \ldots, \boldsymbol{\varphi}^{(N-1)}\right\}
$$

The general expression presented in (14) corresponds to case (e).

\section{Wall Mitigation Techniques}

In this section, we review the front wall clutter mitigation techniques of spatial filtering and subspace projection presented in [28] and [30], respectively.

\section{A. Spatial Filtering Approach}

From (5), we note that $\tau_{w}$ does not vary with the antenna location since the array is parallel to the wall. Furthermore, as the wall is homogeneous and assumed to be much larger than the beamwidth of the antenna, the first term in (5) assumes the same value across the array aperture. Unlike $\tau_{w}$, the time delay $\tau_{p, n}$ in (5) is different for each antenna location, since the signal path from the antenna to the target is different from one antenna to the other. For the $m$ th frequency, the received signal is a function of $n$ via the variable $\tau_{p, n}$. Therefore, we can rewrite (5) as

$$
y_{\omega_{m}}(n)=v_{\omega_{m}}+\sum_{p=0}^{P-1} u_{p, \omega_{m}}(n)
$$

where $v_{\omega_{m}}=\sigma_{w} e^{-j \omega_{m} \tau_{w}}$ and $u_{p, \omega_{m}}(n)=\sigma_{p} e^{-j \omega_{m} \tau_{p, n}}$. Thus, separating wall reflections from target reflections amounts to basically separating constant from non-constant valued signals across antennas, which can be performed by applying a proper spatial filter [28] across the antenna array.

In its simplest form, the spatial filter, which removes, or significantly attenuates, the zero spatial frequency component, can be implemented as the subtraction of the average of the radar return across the antennas. That is

$$
\tilde{y}_{\omega_{m}}(n)=y_{\omega_{m}}(n)-\bar{y}_{\omega_{m}}
$$

where

$$
\bar{y}_{\omega_{m}}=\frac{1}{N} \sum_{n=0}^{N-1} y_{\omega_{m}}(n) .
$$

It can be readily shown that the spatial frequency transform of $\tilde{y}_{\omega_{m}}(n)$ is given by

$$
\begin{aligned}
\tilde{Y}_{\omega_{m}}(\kappa) & =\sum_{n=0}^{N-1} \tilde{y}_{\omega_{m}}(n) \exp (-j 2 \pi \kappa n / N) \\
& =\sum_{n=0}^{N-1}\left(y_{\omega_{m}}(n)-\bar{y}_{\omega_{m}}\right) \exp (-j 2 \pi \kappa n / N) \\
& =Y_{\omega_{m}}(\kappa)-N \bar{y}_{\omega_{m}} \delta[\kappa]
\end{aligned}
$$

where $\kappa$ is the spatial frequency. As $\left.Y_{\omega_{m}}(\kappa)\right|_{\kappa=0}$ equals $N \bar{y}_{\omega_{m}}$, the filtered spatial spectrum $\tilde{Y}_{\omega_{m}}(\kappa)$ is 0 for $\kappa=0$; otherwise, $\tilde{Y}_{\omega_{m}}(\kappa)=Y_{\omega_{m}}(\kappa)$. Thus, the subtraction operation removes the single spatial frequency component $(\kappa=0)$ without changing other components. In practice, the spatial spectrum of the wall reflections may have a non-zero width due to unstable antenna path and/or local inhomogeneities, and a more sophisticated filter with a wider stopband could be applied. However, care should be exercised to not alter the target response. These issues are discussed in detail in [28]. The output spatial filter data will have little or no contribution from the wall reflections.

\section{B. Subspace Projection}

The signals received by the $N$ antennas at the $M$ frequencies are arranged into an $M \times N$ matrix, $\mathbf{Y}$

$$
\mathbf{Y}=\left[\begin{array}{lllll}
\mathbf{y}_{0} & \ldots & \mathbf{y}_{n} & \ldots & \mathbf{y}_{N-1}
\end{array}\right]
$$

where $\mathbf{y}_{n}$ is the $M \times 1$ vector containing the stepped-frequency signal received by the $n$th antenna

$$
\mathbf{y}_{n}=[y(0, n) \ldots y(m, n) \ldots y(M, n)]^{T}
$$


with $y(m, n)$ given by (5). The eigenstructure of the imaged scene is obtained by performing the SVD of $\mathbf{Y}$

$$
\mathbf{Y}=\mathbf{U} \boldsymbol{\Lambda} \mathbf{V}^{H}
$$

where " $\mathrm{H}$ " denotes the Hermitian transpose, $\mathbf{U}$ and $\mathbf{V}$ are unitary matrices containing the left and right singular vectors, respectively, and $\boldsymbol{\Lambda}$ is a diagonal matrix containing the singular values $\lambda_{1}, \lambda_{2}, \ldots, \lambda_{N}$ in decreasing order, i.e.,

$$
\boldsymbol{\Lambda}=\left(\begin{array}{c}
\lambda_{1} \ldots 0 \\
\vdots \ddots \\
0 \ldots \lambda_{N} \\
\vdots \ddots \\
0 \ldots
\end{array}\right)
$$

and $\lambda_{1} \geq \lambda_{2} \geq \ldots \geq \lambda_{N}$. Without loss of generality, the number of frequencies is assumed to exceed the number of antenna locations, i.e., $M>N$.

The subspace projection method assumes that the wall returns and the target reflections lie in different subspaces. Therefore, the first $K$ dominant singular vectors of the $\mathbf{Y}$ matrix are used to construct the wall subspace

$$
\mathbf{S}_{\text {wall }}=\sum_{i=1}^{K} \mathbf{u}_{i} \mathbf{v}_{i}^{H} .
$$

The subspace orthogonal to the wall subspace is

$$
\mathbf{S}_{\text {wall }}^{\perp}=\mathbf{I}-\mathbf{S}_{\text {wall }} \mathbf{S}_{\text {wall }}^{H}
$$

where $\mathbf{I}$ is the identity matrix. To mitigate the wall returns, the data matrix $\mathbf{Y}$ is projected on the orthogonal subspace

$$
\tilde{\mathbf{Y}}=\mathbf{S}_{\text {wall }}^{\perp} \mathbf{Y} .
$$

\section{JOINT WALl Mitigation AND CS FOR TWRI}

In this section, we consider joint wall mitigation and sparsitybased scene reconstruction under the various measurement strategies enumerated in Section III.

\section{A. Same Set of Reduced Frequencies at Each Antenna Location}

Both spatial filtering and subspace projection methods for wall clutter reduction rely on the fact that the wall reflections are the same for all antenna locations. When the same set of frequencies is employed for all employed antennas, i.e., cases (a), (b), and (c), the condition of spatial invariance of the wall reflections is not violated. This permits direct application of the wall clutter mitigation methods as a preprocessing step to the $l_{1}$ norm-based scene reconstruction of (15).

\section{B. Different Set of Reduced Frequencies at Each Antenna Location}

Cases (d) and (e) use different sets of reduced frequencies for the various antenna locations, resulting in different wall reflection phase returns across the antenna elements. This would deprive the wall mitigation algorithms of the underlying assumption of spatial invariance of the wall clutter, thereby rendering the direct application of the wall mitigation methods ineffective. Therefore, cases (d) and (e) require additional data processing before the wall clutter mitigation techniques could be applied. More specifically, the range profile at each antenna location is first reconstructed through $l_{1}$ norm minimization using the reduced frequency set. Then, the Fourier transform of each reconstructed range profile is taken to recover the full frequency data measurements at each antenna location. Direct application of the wall mitigation techniques can then proceed, followed by the scene reconstruction. Mathematically, the problem can be formulated as follows. Assuming the range of interest is divided into $M$ equally spaced gates (range resolution cells), the relationship between the $n$th received signal and the target locations can be expressed as

$$
\mathbf{y}_{n}=\mathbf{A} \mathbf{s}_{n}
$$

where $\mathbf{s}_{n}$ is the discrete range profile corresponding to the $n$th antenna location, $\mathbf{y}_{n}$ is the measured data corresponding to all $M$ frequencies at the $n$th antenna, and $\mathbf{A}$ is an $M \times M$ matrix whose $l$ th column is given by

$$
\mathbf{A}_{l}=\left[e^{-j \omega_{0} \frac{2 l d_{r}}{c}} \ldots e^{-j \omega_{M-1} \frac{2 l d_{r}}{c}}\right]^{T} \quad l=0,1, \ldots, M-1
$$

with $d_{r}=c / 2\left(\omega_{M-1}-\omega_{0}\right)$ being the radar range resolution. Note that the dimension of $\mathbf{s}_{n}$ is equal to the number of range gates, whereas the dimension of $\mathbf{y}_{n}$ is equal to the number of frequencies.

Consider $\breve{\mathbf{y}}_{n}$, which is a vector of length $Q(\ll M)$ consisting of elements chosen from $\mathbf{y}_{n}$ as follows:

$$
\breve{\mathbf{y}}_{n}=\varphi^{(n)} \mathbf{y}_{n}=\varphi^{(n)} \mathbf{A} \mathbf{s}_{n}
$$

where the $Q \times M$ matrix $\varphi^{(n)}$ is constructed in the same manner as in (14). Given $\breve{\mathbf{y}}_{n}$, we can recover $\mathbf{s}_{n}$ by solving the following equation:

$$
\hat{\mathbf{s}}_{n}=\arg \min _{\mathbf{s}_{n}}\left\|\mathbf{s}_{n}\right\|_{l_{1}} \quad \text { subject to } \quad \breve{\mathbf{y}}_{n} \approx \varphi^{(n)} \mathbf{A} \mathbf{s}_{n} .
$$

As in the case of (15), exploitation of the prior knowledge about the standoff distance from the wall can speed up the convergence of the conventional OMP (see the Appendix).

Once the range profile $\hat{\mathbf{s}}_{n}$ has been obtained, we can recover all $M$ frequency measurements at the $n$th antenna location as $\hat{\mathbf{y}}_{n}=\mathbf{A} \hat{\mathbf{s}}_{n}$. Then, the wall mitigation methods can be readily applied. Finally, backprojection can be used to reconstruct the image of the scene [23]. However, since the wall clutter has been suppressed, the $l_{1}$ minimization-based scene reconstruction can be applied, in lieu of backprojection, to improve the target-to-clutter ratio (TCR). 
(a)

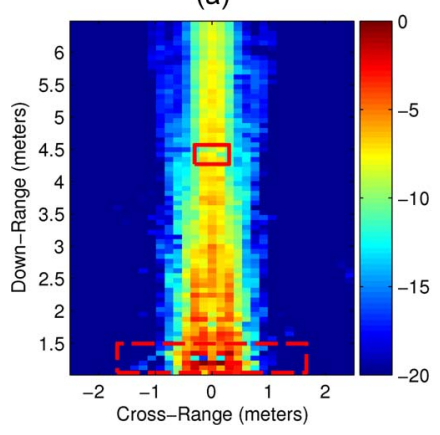

(b)

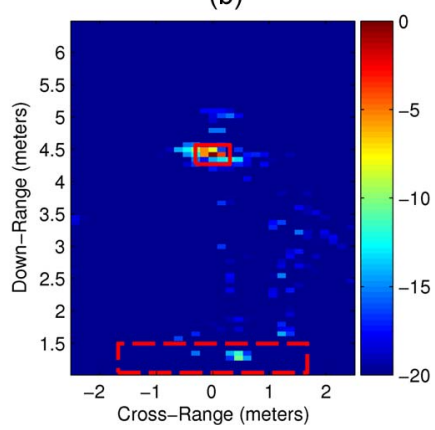

Fig. 2. Backprojection images of the scene. (a) No preprocessing. (b) After background subtraction.

\section{EXPERIMENTAL RESUlts}

\section{A. Experimental Setup}

A through-the-wall wideband synthetic aperture radar system was set up in the Radar Imaging Lab at Villanova University. A 67-element line array with an inter-element spacing of $0.0187 \mathrm{~m}$, located along the $x$-axis, was synthesized parallel to a 0.14-m-thick solid concrete wall of length $3.05 \mathrm{~m}$ and at a standoff distance equal to $1.24 \mathrm{~m}$. A stepped-frequency signal covering the $1-3 \mathrm{GHz}$ frequency band with a step size of $2.75 \mathrm{MHz}$ was employed, providing a range resolution $d_{r}$ of $0.075 \mathrm{~m}$. Thus, at each scan position, the radar collects 728 frequency measurements. A vertical metal dihedral was used as the target and was placed at $(0,4.4) \mathrm{m}$ on the other side of the front wall. The size of each face of the dihedral is $0.39 \mathrm{~m}$ by $0.28 \mathrm{~m}$. The empty scene without the dihedral target present was also measured for comparison. The region to be imaged is chosen to be $4.9 \mathrm{~m} \times 5.4 \mathrm{~m}$ centered at $(0,2.7) \mathrm{m}$ and divided into $33 \times 73$ pixels, respectively. The back and the side walls of the room were covered with RF absorbing material to reduce clutter. However, Section VI-E shows the effectiveness of the proposed techniques for the case where the back wall is not covered with absorbing material.

\section{B. Effect of the Wall Clutter}

Fig. 2(a) shows the image corresponding to the measured scene obtained with backprojection applied directly to the full raw data set. In this figure and all subsequent figures in this paper, we plot the image intensity with the maximum intensity value in each image normalized to $0 \mathrm{~dB}$. The true target position is indicated with a solid red rectangle. With the availability of the empty scene measurements, background subtraction generates an image where the target can be easily identified, as shown in Fig. 2(b). Fig. 3(a) shows the result obtained with $l_{1}$ norm reconstruction using the full raw data set, while the $l_{1}$ norm reconstruction result after background subtraction is provided in Fig. 3(b). The classic OMP was used in both cases. The number of iterations of the OMP is usually associated with the level of sparsity of the scene. For this particular example, the number of OMP iterations was set to 100 for Fig. 3(a) and 5 for Fig. 3(b). Since access to the background scene is not available in practice, it is evident from Fig. 3(a) that the wall mitigation techniques must be applied, as a preprocessing step, prior to CS in order to detect the targets behind the wall. As a (a)

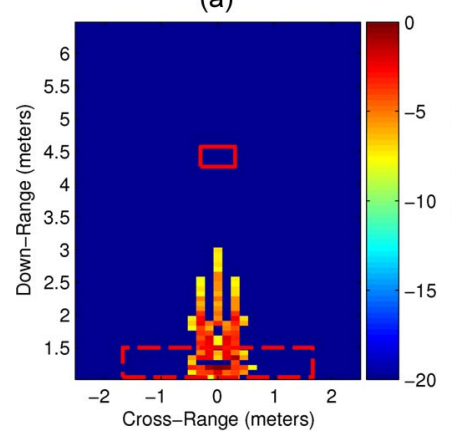

(b)

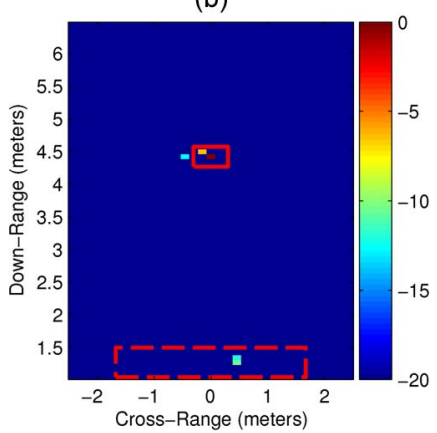

Fig. 3. $l_{1}$ norm reconstruction obtained considering full data set. (a) No preprocessing-classic OMP. (b) After background subtraction-classic OMP.

TABLE I

TCR: BACKGROUND SUBTRACTION-FULL DATA

\begin{tabular}{|c|c|c|}
\hline Clutter Method & Backprojection & CS with OMP \\
\hline Type I & 24.67 & 3072.84 \\
\hline Type II & 24.62 & 9724.16 \\
\hline
\end{tabular}

performance measure, we use the TCR [28], which is defined as the ratio between the maximum pixel magnitude value of the target to the average pixel magnitude value in the clutter region

$$
T C R=20 \log _{10}\left(\frac{\max _{(k, l) \in A_{t}}|r(k, l)|}{\frac{1}{N_{c}} \sum_{(k, l) \in A_{c}}|r(k, l)|}\right)
$$

where $A_{t}$ is the target area, $A_{c}$ is the clutter area, and $N_{c}$ is the number of pixels in the clutter area. The target area is manually selected in close vicinity to the target (9 pixels in all). We consider two types of clutter areas. Type I clutter area is made up of all pixels, excluding the target area and including the wall area, whereas Type II clutter area is the same as Type I, but without considering the wall as a clutter. Figs. 2 and 3 show the target and wall clutter areas, indicated by solid and dashed rectangles, respectively. Table I shows the TCR values for the background subtracted images. As expected, the TCR is improved when using $l_{1}$ reconstruction over backprojection.

\section{Wall Clutter Mitigation}

1) Case (a)_Full Sets of Frequencies and Antenna Locations: The space-frequency sampling pattern corresponding to case (a) is shown in Fig. 4, where the vertical axis represents the antenna location, and the horizontal axis represents the frequency. The filled boxes represent the sampled data. If the full data set of 728 frequencies and 67 antenna locations are available, the preprocessing based on the spatial filtering method defined in (20) and the subspace projection method defined in (29) results in the backprojection images shown in Fig. 5(a) and (b), respectively. In general, the dimension of the wall subspace depends on the wall-type and building material. However, [40] showed that, for a frequency-independent homogeneous wall, the wall subspace is 1-D. Thus, in all subspace projection-based method results presented in this section, the first dominant singular vector $(K=1)$ of the data matrix $\mathbf{Y}$ 


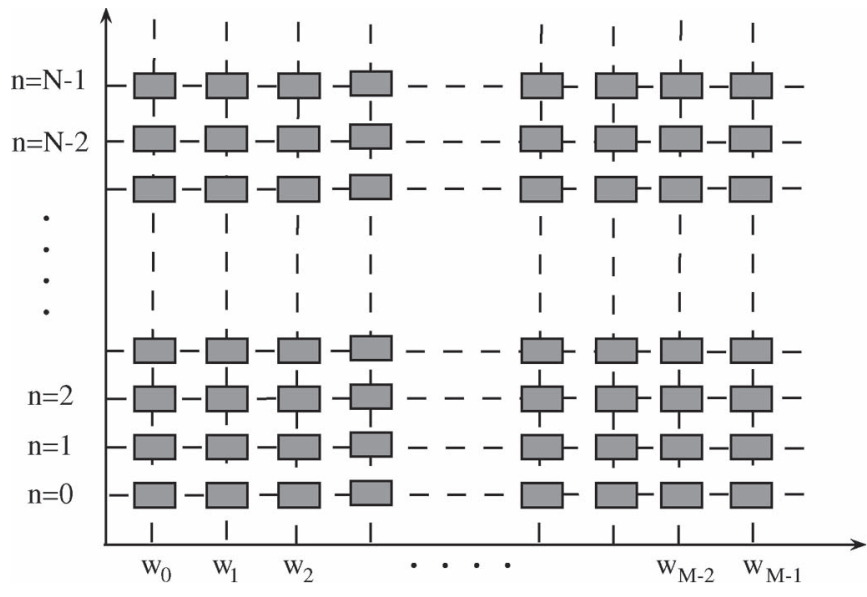

Fig. 4. Case (a)-Illustration of an example of the pattern of the conventional configuration.

(a)
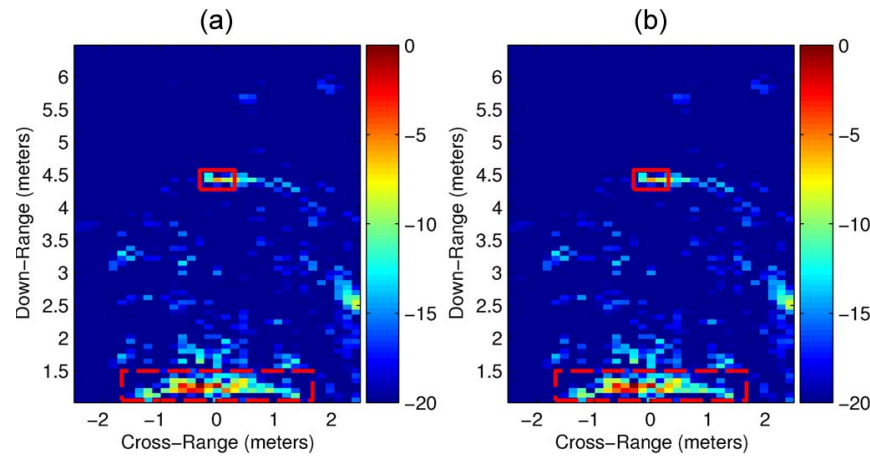

Fig. 5. Case (a) backprojection images. (a) Spatial filtering. (b) Subspace projection.

is used to construct the wall subspace. Although the wall return has not been completely suppressed in both Fig. 5(a) and (b), its shadowing effect has been sufficiently reduced, allowing the detection of the target. Fig. 6(a) and (b) show the $l_{1}$ reconstructed images obtained with the classic OMP for the spatial filtering and the subspace projection-based methods, respectively. Finally, Fig. 6(c) and (d) depict the $l_{1}$ reconstructed images obtained using the modified OMP instead of the classic OMP. The classic OMP required 25 iterations to include the target in the reconstructions in Fig. 6(a) and (b), whereas the scene reconstructions using the modified OMP in Fig. 6(c) and (d) required only five iterations. Therefore, exploitation of the prior knowledge about the standoff distance from the wall resulted in an $80 \%$ reduction in the number of iterations required by the OMP. The TCRs of the aforementioned figures are provided in Table II. We observe from Table II that both classic and modified OMP-based CS reconstructions provide an improvement over the corresponding backprojection results. However, in case of modified OMP, the reduction in the number of iterations is achieved at the expense of the TCR for Type I clutter, which is reduced due to an increase in the wall clutter residuals compared to classic OMP reconstruction. Further, comparing the TCR values in Tables I and II, we observe that, as expected, background subtraction provides higher TCR compared with the wall mitigation results. (a)

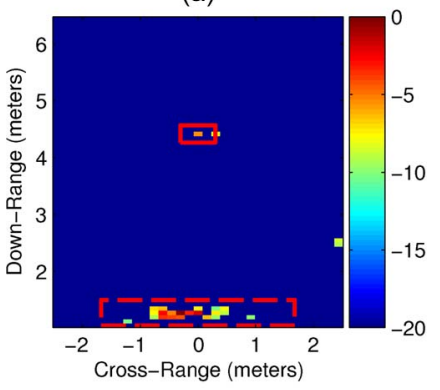

(c)

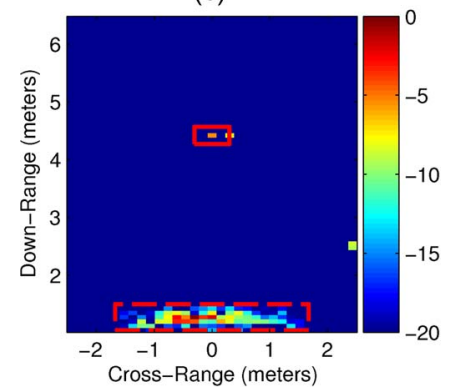

(b)

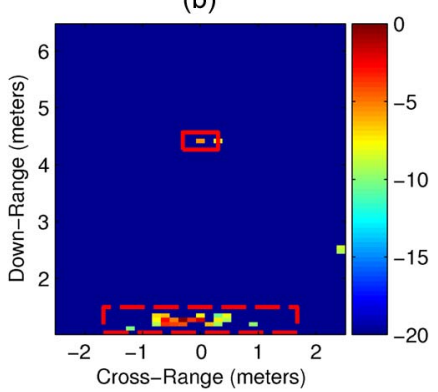

(d)

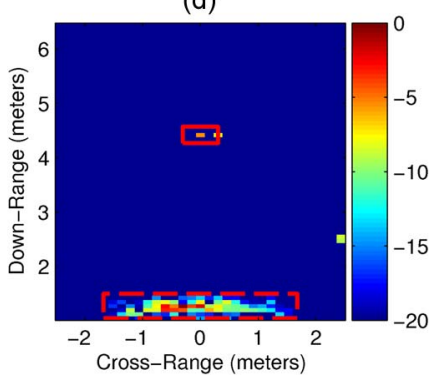

Fig. 6. Case (a) $l_{1}$ reconstruction-based imaging results. (a) Spatial filtering-classic OMP. (b) Subspace projection-classic OMP. (c) Spatial filtering-modified OMP. (d) Subspace projection-modified OMP.

2) Case (b)-Same Reduced Frequency Set for All Antenna Locations: Instead of using full frequency data, we first use 146 uniformly selected frequencies at each of the 67 antenna locations, which represent $20 \%$ of the total data volume. The corresponding space-frequency sampling pattern is shown in Fig. 7(a). Applying the spatial filtering and the subspace projection methods to the reduced data set provides the backprojection images shown in Fig. 8(a) and (b), respectively, which are degraded compared to their CS counterparts, shown in Fig. 9. Both classic OMP and modified OMP were used to recover the scene image using only $20 \%$ of the frequency measurements. The $l_{1}$ minimization-based reconstructed images using classic OMP are plotted in Fig. 9(a) and (b) for the spatial filtering and the subspace projection methods, respectively, where the number of iterations was set to 25 . The corresponding results using the modified OMP with the number of iterations set to 5 are shown in Fig. 9(c) and (d). It is evident that the two wall mitigation techniques maintain their proper performance when the same subset of uniformly selected frequencies is considered at each antenna location. This statement is confirmed by the comparison of the TCR values for case (b), provided in Table III, and the TCR values for case (a) in Table II. Alternatively, the reduced set of frequencies can be chosen randomly instead of a uniform selection, as shown in Fig. 7(b). Backprojection and CS methods are applied to the reduced data comprising $20 \%$ randomly selected spatial-frequency measurements, maintaining the same set of reduced frequencies at each antenna location. In this case, and for all subsequent random selectionbased imaging, each imaged pixel is the result of averaging 100 runs, with a different random selection for each run. The results for the random selection are summarized in Table III. The TCR values provided in Table III suggest that, compared to uniform selection, the random frequency selection reduces the 
TABLE II

TCR: CASE (A)

\begin{tabular}{|c|c|c|c|c|}
\hline Wall mitigation & Clutter & Backprojection & CS with OMP & CS with modified OMP \\
\hline \multirow{2}{*}{ Spatial Filtering } & Type I & 7.62 & 109.11 & 47.44 \\
\cline { 2 - 5 } & Type II & 8.52 & 1097.20 & 1099.30 \\
\hline \multirow{2}{*}{ Subspace projection } & Type I & 7.65 & 109.60 & 47.74 \\
\cline { 2 - 5 } & Type II & 8.54 & 1096.27 & 1098.62 \\
\hline
\end{tabular}
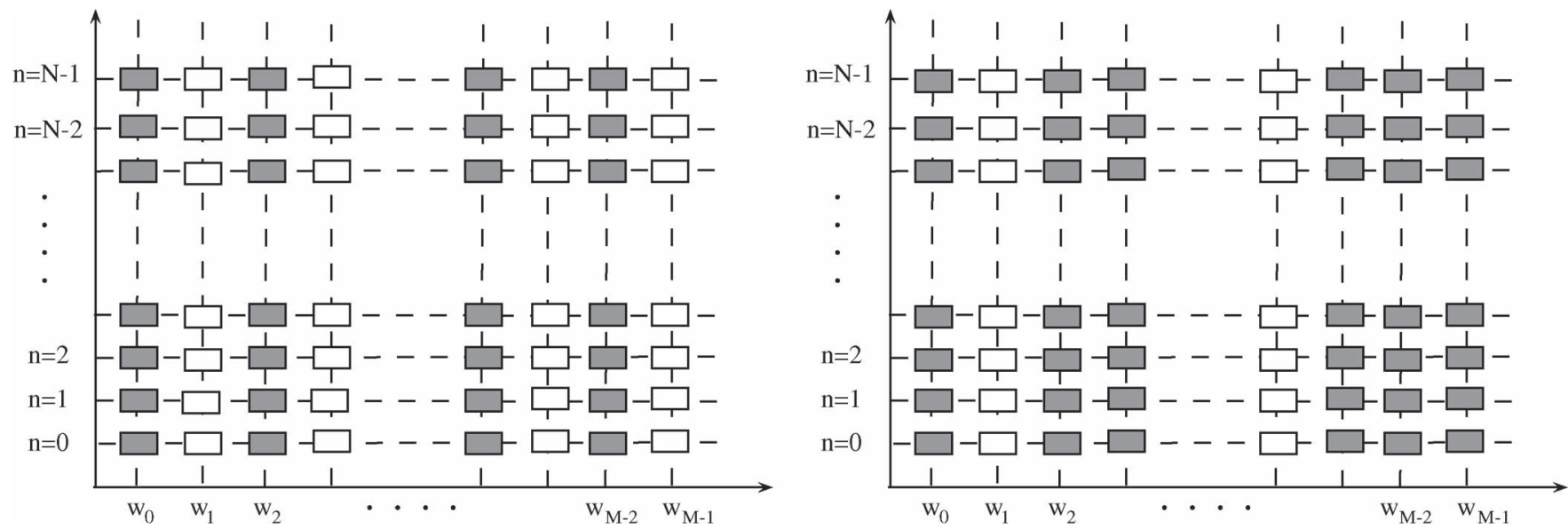

Fig. 7. Case (b) - Illustration of an example of subsampling pattern of the conventional configuration. (a) Uniform subsampling. (b) Random subsampling.

(a)
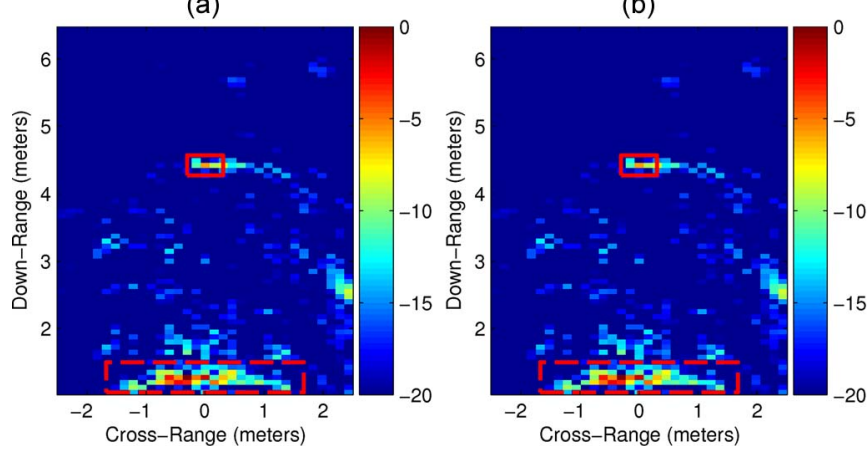

Fig. 8. Case (b) backprojection images with uniformly selected frequencies (a) Spatial filtering. (b) Subspace projection.

quality of the backprojection images while preserving the CS reconstructed image quality. Similar to the uniform selection, the performance of the modified OMP is comparable to that of the classic OMP for Type II clutter, while it degrades for Type I clutter.

3) Case (c)-Same Set of Reduced Frequencies for a Reduced Set of Antenna Locations: The space-frequency sampling pattern for case (c) is shown in Fig. 10(a) for uniform sampling and in Fig. 10(b) for random sampling. We first consider 146 uniformly selected frequencies (20\% of 728) and 34 uniformly selected array locations (51\% of 67), which collectively represent $10.2 \%$ of the total data volume. As in case (b), spatial filtering is implemented by subtraction of the average value of (20) across the thinned array for each of the reduced number of frequencies. The subspace projection-based method is applied, in this case, to a reduced dimension $\mathbf{Y}$ matrix, (a)

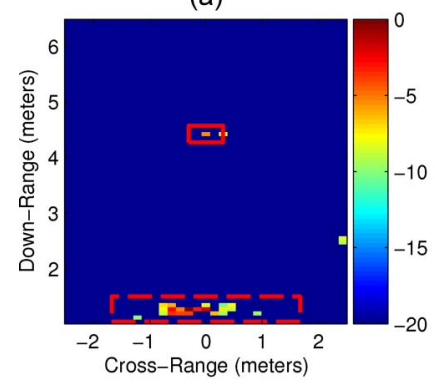

(c)

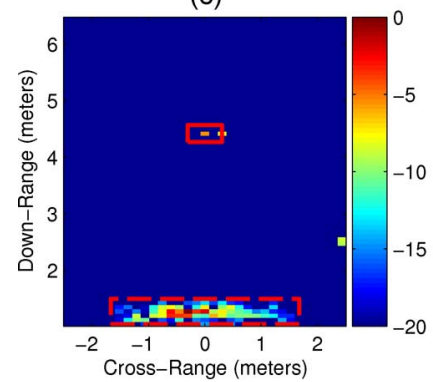

(b)

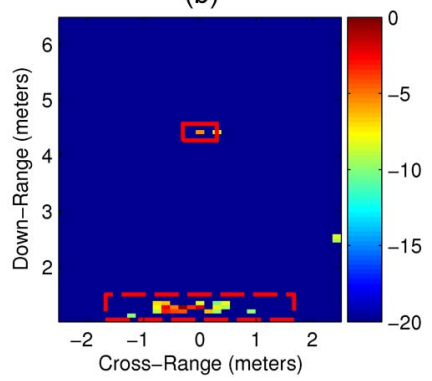

(d)

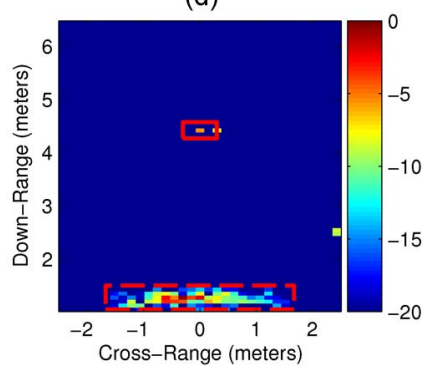

Fig. 9. Case (b) $l_{1}$ reconstruction-based imaging results. (a) Spatial filtering-classic OMP. (b) Subspace projection-classic OMP. (c) Spatial filtering —-modified OMP. (d) Subspace projection—-modified OMP.

$146 \times 34$ instead of $146 \times 67$ for case (b) and $728 \times 67$ for case (a). The backprojection images corresponding to spatial filtering and subspace projection methods are shown in Fig. 11(a) and (b), respectively. The $l_{1}$ norm reconstructed images obtained with classic OMP are shown in Fig. 12(a) and (b) for the spatial filter and subspace projection approaches, respectively, which have less wall clutter residuals 
TABLE III

TCR: CASE (B)

\begin{tabular}{|c|c|c|c|c|c|}
\hline Frequency Selection & Wall mitigation & Clutter & Backprojection & CS with OMP & CS with modified OMP \\
\hline \multirow{2}{*}{ Uniform } & Spatial Filtering & Type I & 7.53 & 108.56 & 47.02 \\
\cline { 3 - 6 } & & Type II & 8.41 & 1079.60 & 1085.30 \\
\cline { 2 - 6 } & \multirow{2}{*}{ Subspace projection } & Type I & 7.55 & 108.72 & 47.24 \\
\cline { 3 - 6 } & & Type II & 8.43 & 1079.13 & 1085.36 \\
\hline \multirow{2}{*}{ Random } & \multirow{2}{*}{ Spatial Filtering } & Type I & 5.00 & 116.44 & 43.30 \\
\cline { 3 - 6 } & & Type II & 5.28 & 943.15 & 1025.57 \\
\cline { 3 - 6 } & \multirow{2}{*}{ Subspace projection } & Type I & 5.06 & 116.71 & 43.55 \\
\cline { 3 - 6 } & & Type II & 5.34 & 944.19 & 1016.08 \\
\hline
\end{tabular}
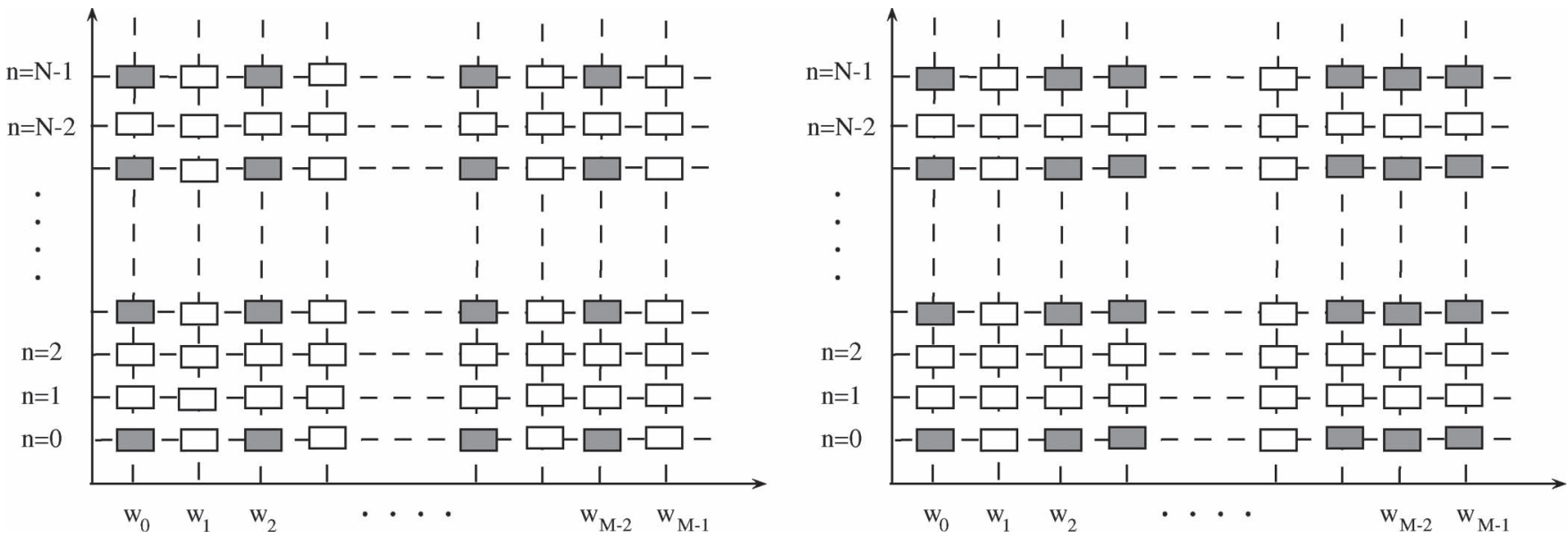

Fig. 10. Case (c) - Illustration of an example of subsampling pattern of the conventional configuration. (a) Uniform subsampling. (b) Random subsampling.

(a)
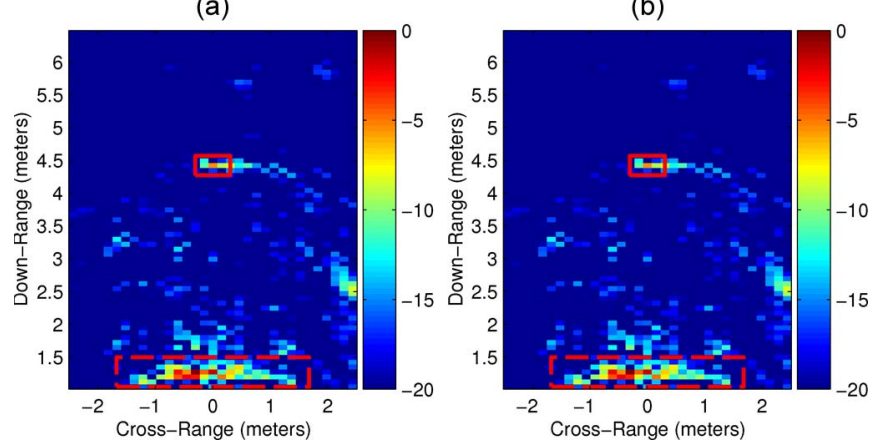

Fig. 11. Case (c) backprojection images. (a) Spatial filtering. (b) Subspace projection.

compared to the corresponding modified OMP images, shown in Fig. 12(c) and (d). It is clear that, even when both spatial and frequency observations are reduced, the CS results for both classic and modifed OMP provide better and cleaner images than those using backprojection. The advantage of CS over backprojection is evident in Table IV, where the TCR for the uniform sampling are illustrated. As expected, the performance of the modified OMP is comparable to that of the classic OMP for Type II clutter, while it degrades for Type I clutter. The important point to note here is that the (a)

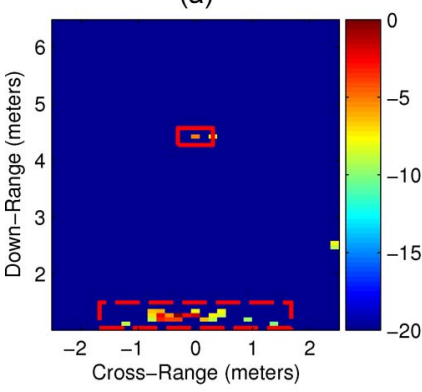

(c)

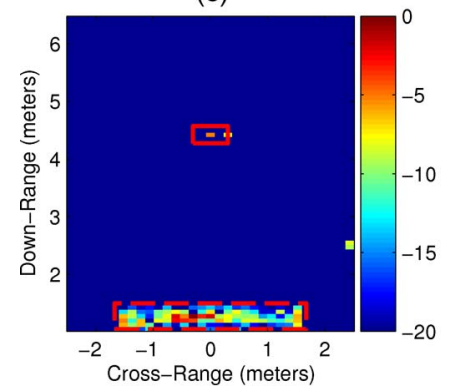

(b)

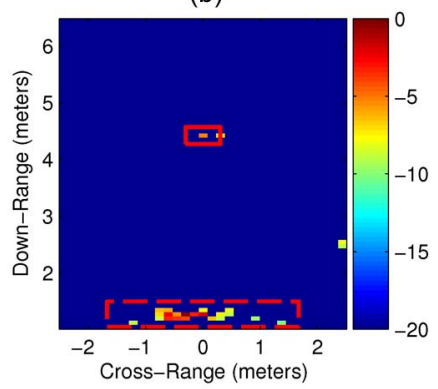

(d)

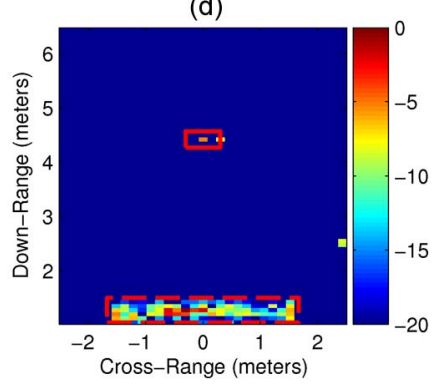

Fig. 12. Case (c) $l_{1}$ reconstruction-based imaging results. (a) Spatial filtering-classic OMP. (b) Subspace projection-classic OMP. (c) Spatial filtering—-modified OMP. (d) Subspace projection—-modified OMP. 
TABLE IV

TCR: CASE (C)

\begin{tabular}{|c|c|c|c|c|c|}
\hline Frequency Selection & Wall mitigation & Clutter & Backprojection & CS with OMP & CS with modified OMP \\
\hline \multirow{3}{*}{ Uniform } & Spatial Filtering & Type I & 7.64 & 109.60 & 39.01 \\
\cline { 3 - 6 } & & Type II & 8.53 & 1085.22 & 1101.21 \\
\cline { 2 - 6 } & \multirow{2}{*}{ Subspace projection } & Type I & 7.66 & 109.66 & 39.13 \\
\cline { 3 - 6 } & & Type II & 8.55 & 1085.34 & 1101.78 \\
\hline \multirow{2}{*}{ Random } & \multirow{2}{*}{ Spatial Filtering } & Type I & 4.12 & 94.36 & 29.65 \\
\cline { 3 - 6 } & & Type II & 4.35 & 767.17 & 808.01 \\
\cline { 3 - 6 } & \multirow{2}{*}{ Subspace projection } & Type I & 4.23 & 98.75 & 32.04 \\
\cline { 3 - 6 } & & Type II & 4.47 & 851.08 & 877.10 \\
\hline
\end{tabular}

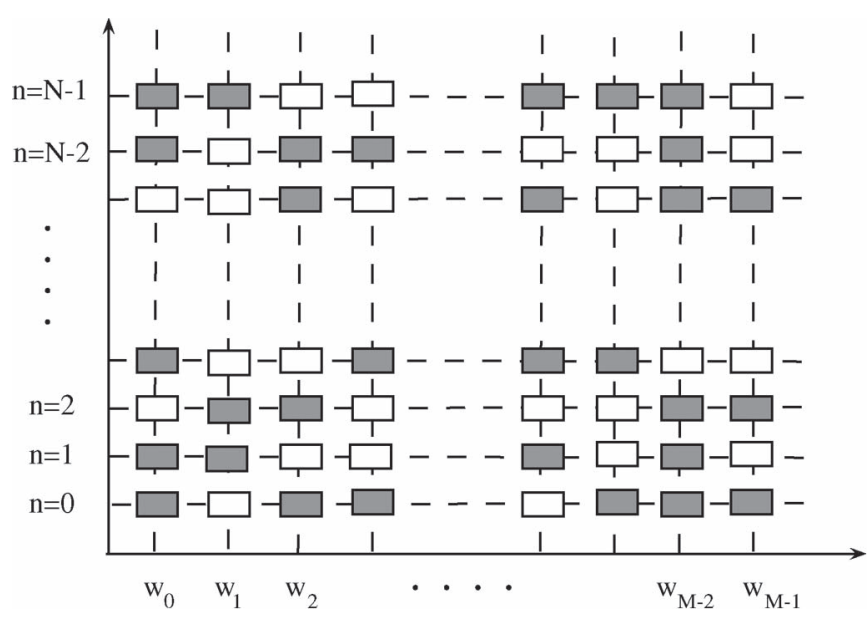

Fig. 13. Case (d) - Illustration of an example of random subsampling pattern of the conventional configuration.

direct application of wall mitigation methods is robust to the reduction of the spatial domain samples. The random spatialfrequency measurements of Fig. 10(b) can alternatively be used for CS-based imaging. The corresponding TCR values are listed in Table IV, which indicate that, similar to the uniform selection, the performance of the modified OMP is comparable to that of the classic OMP for Type II clutter, while it degrades for Type I clutter under random selection.

4) Case (d)_Different Sets of Reduced Frequencies for All Antenna Locations: As discussed in the introduction, having the same frequency observations may not always be possible. Use of different sets of reduced frequencies at different antenna locations would lead to different wall reflection phase returns across the antennas, rendering the wall mitigation methods ineffective. The method proposed in Section V-B combats this shortcoming by recovering all the frequency measurements at each antenna location through $l_{1}$ norm range profile reconstruction. Both classic OMP and modified OMP were used to recover the range profiles using only $20 \%$ of the frequency measurements. The space-frequency sampling pattern for case (d) is shown in Fig. 13. Fig. 14(a) and (c) shows the backprojection images obtained after applying spatial filtering to the full data recovered from the reconstructed range profiles using classic OMP and modified OMP, respectively. Fig. 14(b) (a)

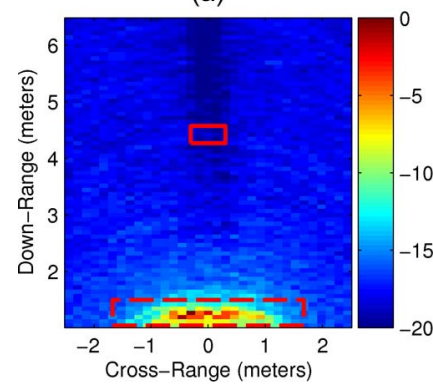

(c)
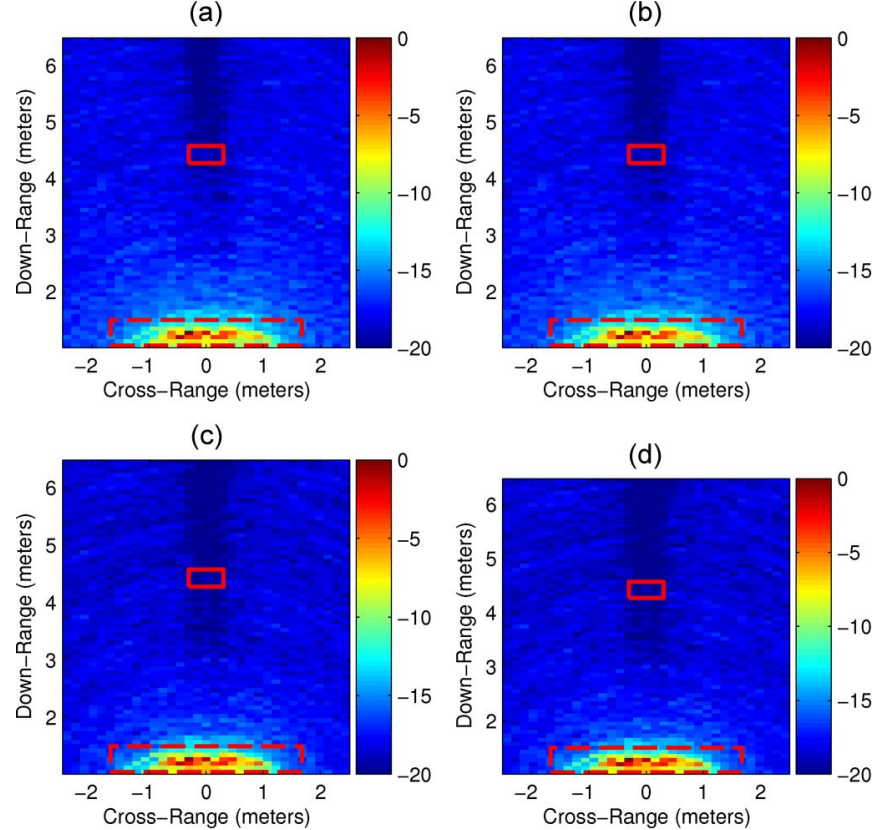

(d)

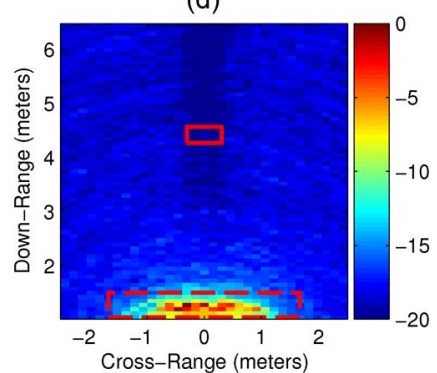

Fig. 14. Case (d) backprojection images with $20 \%$ frequency measurements. (a) Spatial filtering-range profiles with classic OMP. (b) Subspace projection-range profiles with classic OMP. (c) Spatial filtering-range profiles with modified OMP. (b) Subspace projection-range profiles with modified OMP.

and (d) shows the corresponding results when the subspace projection method was used for wall clutter mitigation. We observe that the target has not been localized in these images, which is in contrast to case (b), wherein target localization was successfully achieved with $20 \%$ data volume. This is because, in case (d), the various range profiles are reconstructed prior to the application of wall clutter mitigation techniques. As the sparsity in range is lower in the presence of wall clutter, the number of ob served frequencies required for sparse reconstruction of the range profiles is expected to be higher compared with case (b). Next, the number of frequencies used for range profile reconstruction was increased to $40 \%$. The corresponding backprojection images for classic OMP and modified OMP based range profile reconstructions are provided in Fig. 15(a) and (c), respectively, for the spatial filtering approach, and in Fig. 15(b) and (d), respectively, for the subspace projection. 
(a)

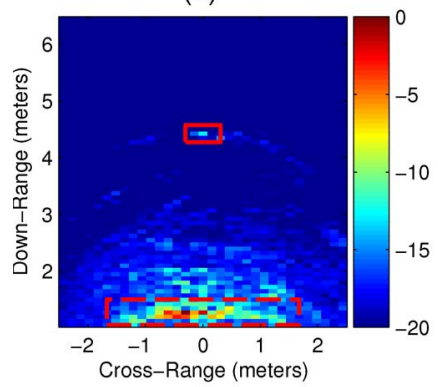

(c)
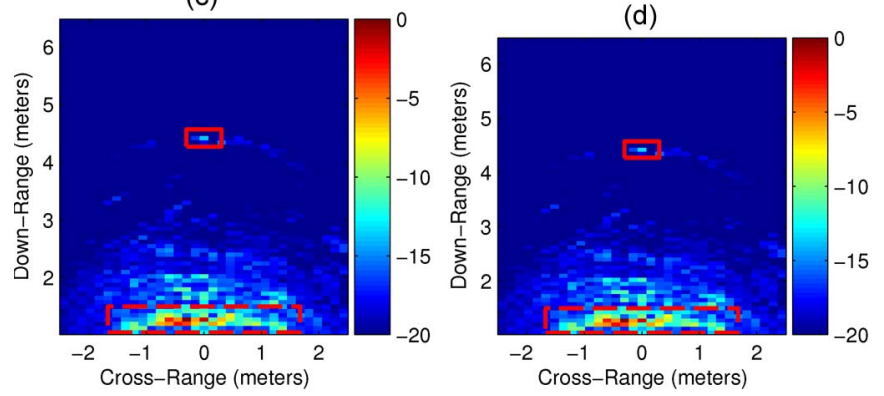

Fig. 15. Case (d) backprojection images with $40 \%$ frequency measurements. (a) Spatial filtering-range profiles with classic OMP. (b) Subspace projection-range profiles with classic OMP. (c) Spatial filtering-range profiles with modified OMP. (b) Subspace projection-range profiles with modified OMP

TABLE V

TCR: CASE (D)—BACKPROJECTION USING $l_{1}$ ReCONSTRUCted Range Profiles

\begin{tabular}{|c|c|c|c|}
\hline Wall mitigation & Clutter & classic OMP & modified OMP \\
\hline \multirow{2}{*}{ Spatial Filtering } & Type I & 2.27 & 2.25 \\
\cline { 2 - 4 } & Type II & 2.47 & 2.48 \\
\hline \multirow{2}{*}{ Subspace projection } & Type I & 2.29 & 2.27 \\
\cline { 2 - 4 } & Type II & 2.49 & 2.50 \\
\hline
\end{tabular}

The classic OMP required 100 iterations for each range profile reconstruction. On the other hand, the modified OMP needed only 70 iterations as all range gates from $0 \mathrm{~m}$ to $1.4 \mathrm{~m}$ were reconstructed in the first iteration, thereby providing a $30 \%$ reduction in the number of iterations compared to classic OMP. The backprojection images for both classic and modied OMPbased results appear very similar. This observation is confirmed by the corresponding TCRs provided in Table V. Comparing the TCR values in Table $\mathrm{V}$ with the backprojection TCR values in Table III under random frequency selections for case (b), we note that the cost for not using the same set of reduced frequency measurements across all antennas manifests itself in terms of a reduced TCR. This loss in quality is due to the fact that the individual range profile reconstructions are not perfect. As a result, there are discrepancies in the recovered full-frequency data compared to the measured full-frequency data, as demonstrated in Figs. 16 and 17 for the first and the middle antenna positions, respectively. Finally, the $l_{1}$ norm image reconstruction was performed, in lieu of backprojection, on the full frequency data recovered from the modified OMPbased reconstructed range profiles. Fig. 18(a) and (b) shows (a)

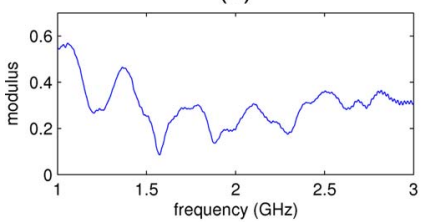

(b)
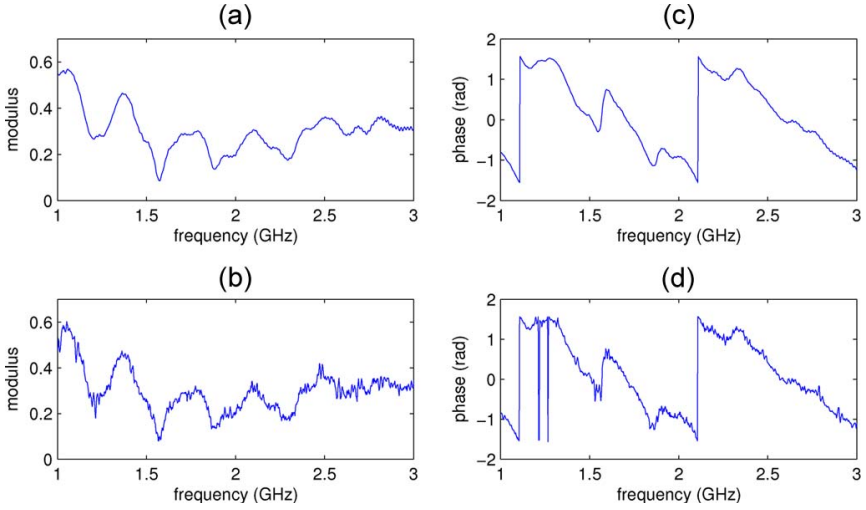

(d)

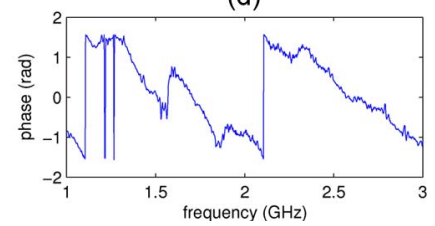

Fig. 16. Comparison between the original data vector $\mathbf{y}_{1}$ and the recovered data vector from the range profile $\hat{\mathbf{y}}_{1}$. (a) Modulus of $\mathbf{y}_{1}$. (b) Modulus of $\hat{\mathbf{y}}_{1}$. (c) Phase of $\mathbf{y}_{1} \cdot$ (d) Phase of $\hat{\mathbf{y}}_{1}$. (a)

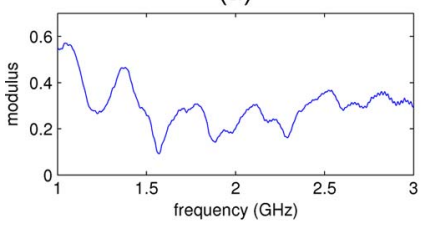

(b)

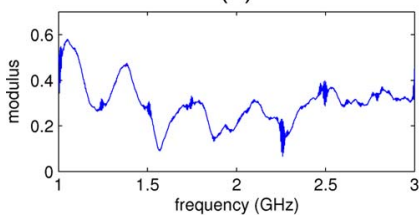

(c)

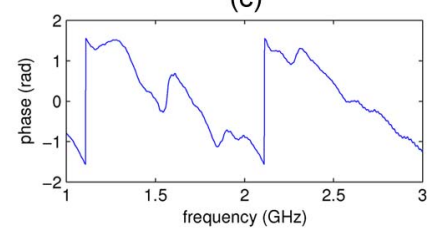

(d)

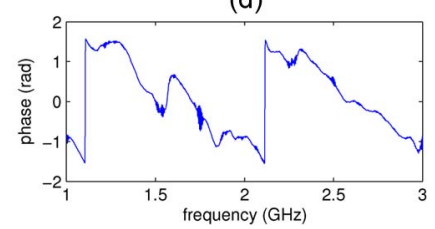

Fig. 17. Comparison between the original data vector $\mathbf{y}_{34}$ and the recovered data vector from the range profile $\hat{\mathbf{y}}_{34}$. (a) Modulus of $\mathbf{y}_{34}$. (b) Modulus of $\hat{\mathbf{y}}_{34}$. (c) Phase of $\mathbf{y}_{34}$. (d) Phase of $\hat{\mathbf{y}}_{34}$.

the images reconstructed using classic OMP corresponding to spatial filtering and subspace projection methods, respectively. Fig. 18(c) and (d) shows the $l_{1}$ reconstruction through modified OMP for spatial filtering and subspace reconstructionbased preprocessing, respectively. In this case, the classic OMP required 100 iterations to ensure the inclusion of the target into the reconstructed image, while the modified OMP needed 70 iterations to achieve the same objective. We observe from Fig. 18 that both classic OMP and modified OMP are able to localize the target. The image quality of the $l_{1}$ reconstructions are compared in Table VI by means of the TCR, which approximately assumes the same values for classic and modified OMP. To reiterate, the penalty for not using the same set of reduced frequency measurements across the antennas is two-fold. First, the reduction in data volume is lower compared to that of case (b) in which the same set of reduced frequencies is employed at each antenna location. Second, despite the use of a larger amount of data in case (d), the corresponding TCR is lower than that for case (b).

5) Case (e)-Different Sets of Reduced Frequencies for a Reduced Set of Antenna Locations: This is the most general case, where both spatial and frequency measurements are reduced and the employed frequency sets differ from one antenna location to the other. The corresponding space-frequency sampling pattern is shown in Fig. 19. We randomly selected $51 \%$ of the antenna locations and randomly chose $40 \%$ of the frequencies at each selected antenna location. Therefore, the 
(a)

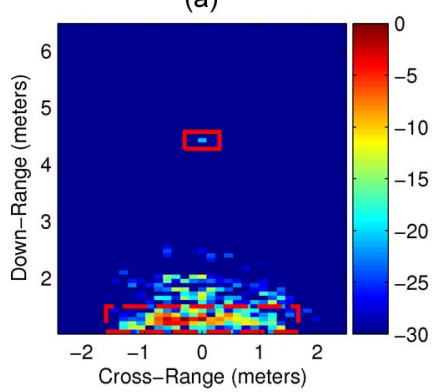

(c)

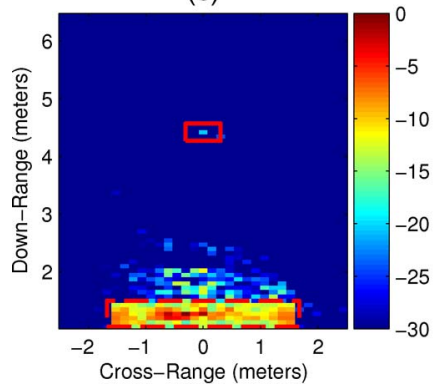

Fig. 18. Case (d) $l_{1}$ reconstruction-based imaging results obtained using the range profiles with modified OMP. (a) Spatial filtering-classic OMP (b) Subspace projection—classic OMP. (c) Spatial filtering-modified OMP. (d) Subspace projection-modified OMP.

TABLE VI

TCR: CASE (D) $-l_{1}$ RECONSTRUCTED IMAGES USING THE RANGe PRofile ObTAINed With Modified OMP

\begin{tabular}{|c|c|c|c|}
\hline Wall mitigation & Clutter & classic OMP & modified OMP \\
\hline \multirow{2}{*}{ Spatial Filtering } & Type I & 5.96 & 3.98 \\
\cline { 2 - 4 } & Type II & 13.01 & 10.88 \\
\hline \multirow{2}{*}{ Subspace projection } & Type I & 6.16 & 4.38 \\
\cline { 2 - 4 } & Type II & 13.41 & 11.96 \\
\hline
\end{tabular}

reduced data set considered in this case represents $20.3 \%$ of the total data volume. Full frequency data measurements were first recovered from both the classic OMP and modified OMPbased reconstructed range profiles at each considered antenna location. Wall mitigation methods were then applied, followed by backprojection for image formation. The corresponding backprojection images for classic OMP and modified OMP are provided in Fig. 20(a) and (c), respectively, for the spatial filtering approach, and in Fig. 20(b) and (d), respectively, for the subspace projection approach. We observe that, despite the use of fewer antenna locations than case (d), the target has been successfully localized in all images. The TCRs of the backprojection images in Fig. 20, summarized in Table VII, indicate a slight deterioration in image quality compared to that under case (d). Next, the $l_{1}$ norm reconstruction procedure was employed for image formation using the full frequency data measurements recovered through modified OMP at the selected antennas. Fig. 21(a) and (b) shows the images reconstructed using classic OMP corresponding to spatial filtering and subspace projection methods, respectively, while Fig. 21(c) and (d) shows the $l_{1}$ reconstruction through modified OMP for spatial filtering and subspace reconstruction-based preprocessing, respectively.

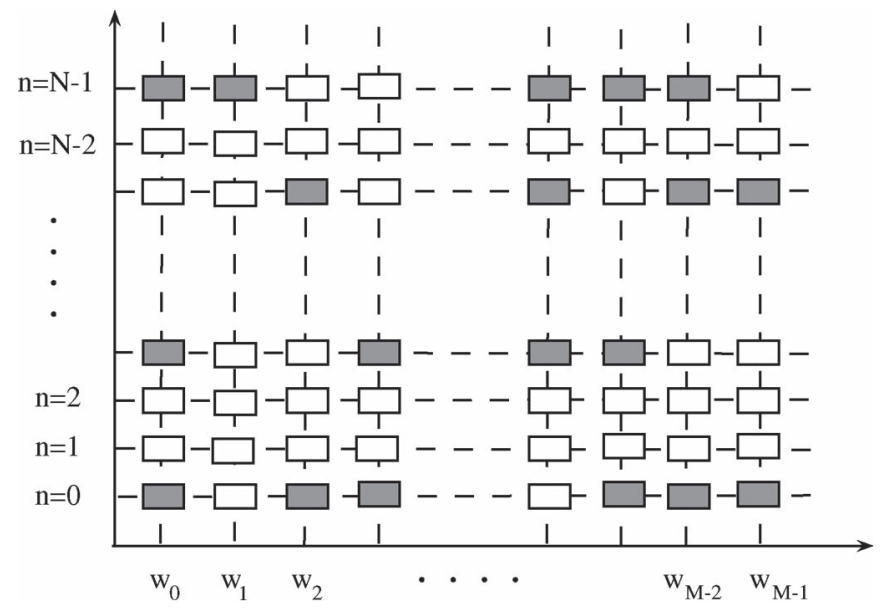

Fig. 19. Case (e) - Illustration of an example of random subsampling pattern of the conventional configuration. (a)

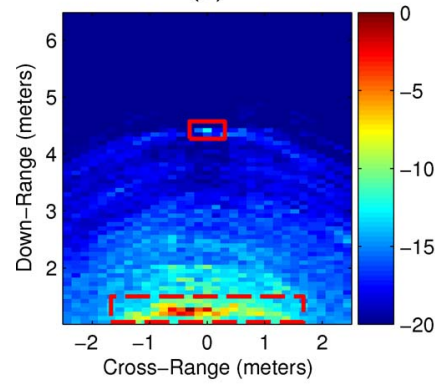

(c)

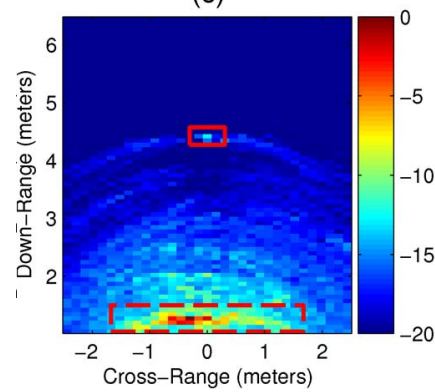

(b)

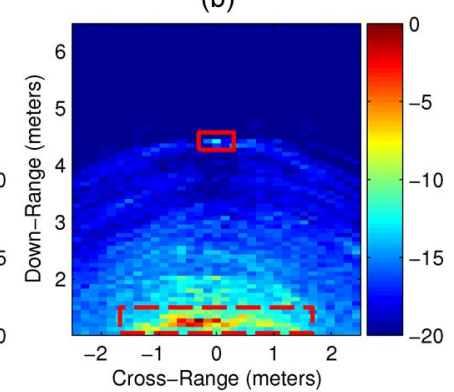

(d)

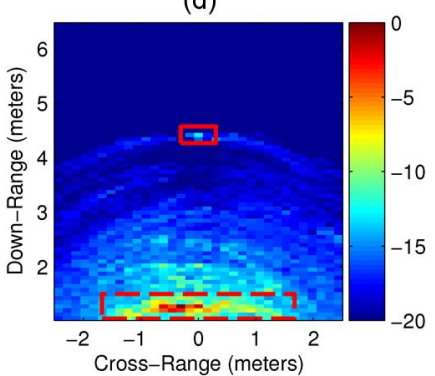

Fig. 20. Case (e) backprojection images with $40 \%$ frequency measurements. (a) Spatial filtering-range profiles with classic OMP. (b) Subspace projection-range profiles with classic OMP. (c) Spatial filtering-range profiles with modified OMP. (d) Subspace projection-range profiles with modified OMP.

Similar to case (d), modified OMP provides a $30 \%$ reduction in the number of iterations required for image reconstruction. The target is visible in both classic and modified OMP-based reconstructed images, albeit with relatively more residual wall clutter for the modified OMP recovered image. These observations are validated by the corresponding TCR values provided in Table VIII.

\section{Summary of the Results}

A recapitulation of the experimental results is provided in this section. The first part of the experimental results showed that the front wall, which is a highly reflective and attenuative medium, presents the main difficulty in revealing behind-thewall stationary targets. Background subtraction achieves the 
TABLE VII

TCR: CASE (E)-BACKPROJECTION USING $l_{1}$ RECONSTRUCTED RANGE PROFILES

\begin{tabular}{|c|c|c|c|}
\hline Wall mitigation & Clutter & classic OMP & modified OMP \\
\hline Spatial Filtering & Type I & 1.80 & 1.88 \\
\cline { 2 - 4 } & Type II & 1.94 & 2.04 \\
\hline Subspace projection & Type I & 1.81 & 1.89 \\
\cline { 2 - 4 } & Type II & 1.96 & 2.06 \\
\hline
\end{tabular}

(a)

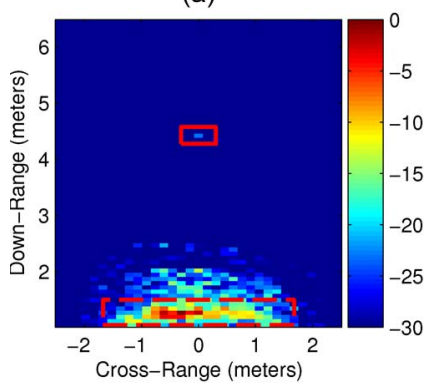

(c)

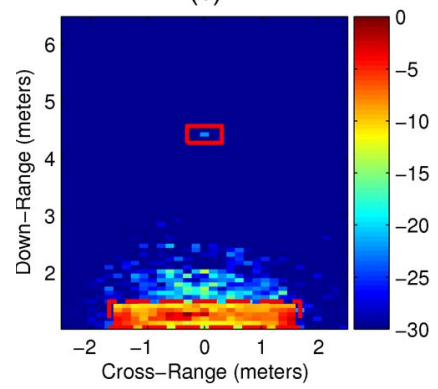

(b)

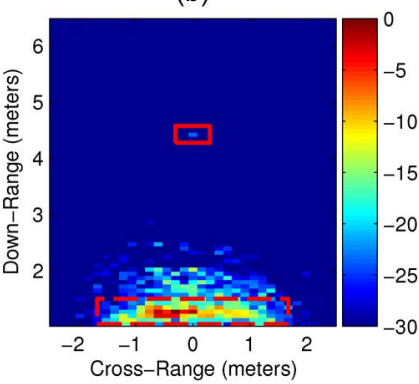

(d)

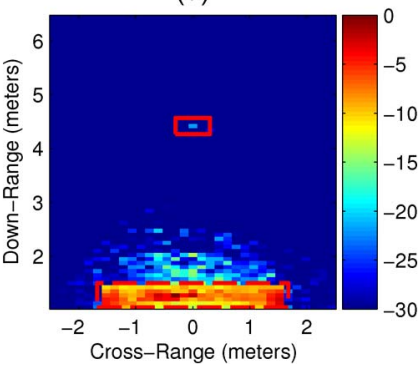

Fig. 21. Case (e) $l_{1}$ reconstruction-based imaging results obtained using the range profiles with modified OMP. (a) Spatial filtering-classic OMP. (b) Subspace projection-classic OMP. (c) Spatial filtering-modified OMP. (d) Subspace projection-modified OMP.

TABLE VIII

TCR: CASE (E) $-l_{1}$ RECONSTRUCTED IMAGES USING THE RANGe Profile OBtained With Modified OMP

\begin{tabular}{|c|c|c|c|}
\hline Wall mitigation & Clutter & classic OMP & modified OMP \\
\hline \multirow{2}{*}{ Spatial Filtering } & Type I & 3.50 & 2.43 \\
\cline { 2 - 4 } & Type II & 6.83 & 6.75 \\
\hline \multirow{2}{*}{ Subspace projection } & Type I & 3.37 & 2.51 \\
\cline { 2 - 4 } & Type II & 6.55 & 6.93 \\
\hline
\end{tabular}

best results in removing wall returns. It provides the highest TCR (24.6 dB in backprojection image) compared with the spatial filtering, and the subspace projection wall mitigation results, which for the non-compressive case, provide TCR of approximately $8 \mathrm{~dB}$ in backprojection images. Access to the background scene is not typically available in practice. Thus, spatial filtering, subspace projection, or any other wall mitigation technique must be applied. The second part of the experimental results dealt with the front wall clutter mitigation in conjunction with CS. The results for both spatial filtering and (a)

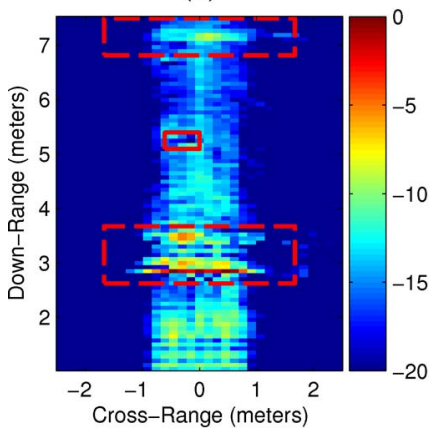

(b)

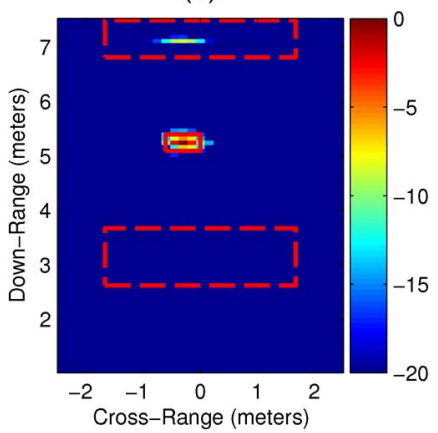

Fig. 22. Backprojection images of the scene without absorbing material in the back wall of the room. (a) No preprocessing. (b) After background subtraction.

subspace projection methods demonstrated that target visibility and high TCR can be achieved when the same subset of frequencies is considered at each antenna location. This is due to the fact that the condition of spatial invariance of the wall reflections required by both spatial filtering and subspace projection methods is not violated when the same set of frequencies is employed at each antenna location. Occasionally, the same operating frequencies might not be available at each antenna position. In this case, the proposed preprocessing step permits the subset of frequencies to differ from one antenna location to another. However, this is accomplished at the expense of target visibility and TCR degradation; the latter being approximately $2 \mathrm{~dB}$ in backprojection images.

\section{E. Effect of the Back Wall Reflections}

In order to demonstrate the robustness of the proposed methods in a less controlled environment, a new through-the-wall experiment was set up in the Radar Imaging Lab at Villanova University. In this experiment, the side walls were covered with the absorbing material, but the 0.31-m-thick reinforced concrete back wall was left uncovered. For imaging, a 93element linear array with an interelement spacing of $0.02 \mathrm{~m}$ was used, which was synthesized using a single horn antenna. The scene was illuminated with a stepped frequency signal of 2-GHz bandwidth centered at $2 \mathrm{GHz}$, using 641 frequencies with a step size of $3.125 \mathrm{MHz}$. A 0.2 -m-thick solid concrete block wall was placed $3.13 \mathrm{~m}$ in front of and parallel to the antenna baseline. The distance between the back face of the front wall and the front of the back wall is $3.76 \mathrm{~m}$. The same metal dihedral used for the previous experiments was used as a target and was placed at $(0.3,5.2) \mathrm{m}$. The region to be imaged is chosen to be $4.9 \mathrm{~m} \times 6.4 \mathrm{~m}$ centered at $(0,4.3) \mathrm{m}$ and divided into $33 \times 87$ pixels, respectively. The backprojected images using the full data measurements are shown in Fig. 22. In Fig. 22(a), no preprocessing was applied to remove the front wall reflections, whereas in Fig. 22(b), background subtraction was applied. Fig. 22 confirms the need to reduce the front wall clutter in order to detect the presence of the target. Next, we applied the same space-frequency sampling pattern for case (c), which is shown in Fig. 10(a). There are 65 uniformly selected frequencies (20\% of 641) and 47 uniformly selected array locations (51\% of 93). The backprojection images corresponding to spatial filtering and subspace projection methods are shown in Fig. 23(a) and (b), respectively. They demonstrate that both 
(a)

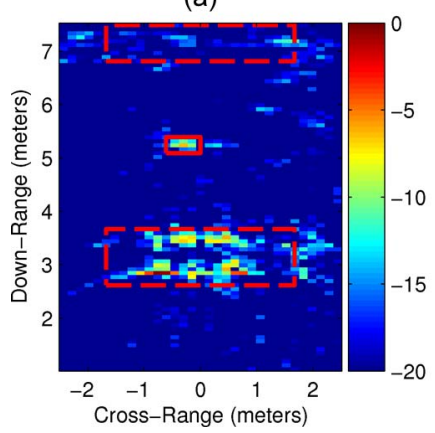

(b)

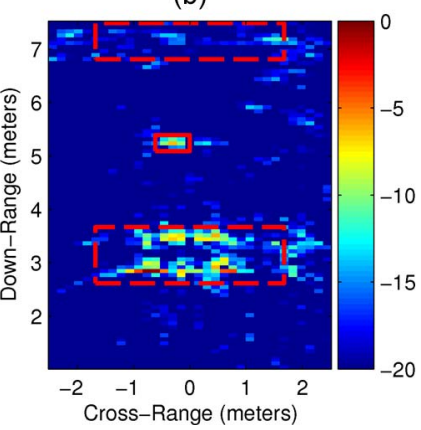

Fig. 23. Case (c) backprojection images of the scene without absorbing material in the back wall of the room. (a) Spatial filtering. (b) Subspace projection. (a)

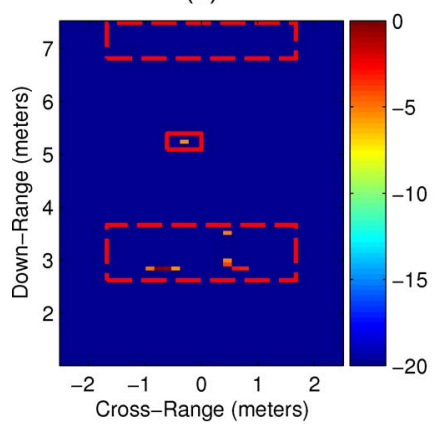

(b)

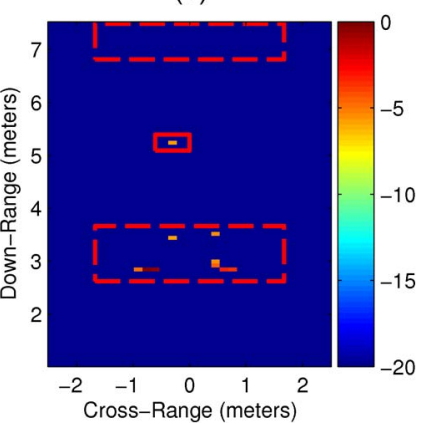

Fig. 24. Case (c) $l_{1}$ reconstruction-based imaging results of the scene without absorbing material in the back wall of the room. (a) Spatial filtering-classic OMP. (b) Subspace projection-classic OMP.

approaches are effective in reducing the wall reflections without significantly compromising the target image. The $l_{1}$ norm reconstructed images obtained with classic OMP are shown in Fig. 24(a) and (b) for the spatial filter and subspace projection approaches, respectively, which have less wall clutter residuals compared to the corresponding backprojected images.

\section{CONCLUSION}

In this paper, we examined the performance of joint mitigation of the wall backscatterings and sparse scene reconstruction in TWRI applications. We focused on two leading methods for combating wall clutter, namely, spatial filtering and subspace projections. Using real data, collected in a laboratory environment with a stepped-frequency radar, we showed that these two methods maintain proper performance when acting on reduced data measurements. We considered two cases of frequency measurement distributions over antennas. Direct application of wall clutter mitigation methods was effective when the same frequencies were used for each antenna. For the cases where different frequencies were used at different antennas, we reconstructed the sparse range profiles with a CS approach applied at each antenna. The signal responses of all frequencies were then generated using the Fourier transform. In so doing, signal processing techniques, such as spatial filtering and subspace projections, can be applied to capture and remove the wall EM returns. The proposed method is flexible in the sense that it permits the use of a different set of frequencies at each antenna location. Subsequent sparse reconstruction using the much reduced wall-clutter data successfully detected and accurately localized the targets. In applying CS, we used prior knowledge of the standoff distance from the wall to speed up the convergence of the OMP for sparse data reconstruction.

\section{APPENDIX \\ MODIFIED OMP}

Prior knowledge of the wall standoff distance can be incorporated into the $l_{1}$ reconstruction algorithm to achieve fast convergence. More specifically, the classic OMP is modified to allow the algorithm to reconstruct all pixels in the image up to and including the wall, in a single iteration. The proposed modified OMP is described in detail in Algorithm 1, where the variables $\Gamma, \bar{Q}$, and $D$ are equivalent to $\Phi \Psi, Q_{1} Q_{2}$, and $N_{x} N_{z}$, defined in Section III-B, respectively. Let the set of columns of the matrix $\Gamma$ corresponding to the pixels up to the wall maximum downrage distance be denoted by $\chi_{\text {wall }}$ and the corresponding set of column indices be $\boldsymbol{\lambda}_{\text {wall }}$. The first iteration in Algorithm 1 reconstructs all of the pixels corresponding to $\chi_{\text {wall }}$. In each subsequent iteration, the algorithm proceeds as for classic OMP. In so doing, the modified OMP algorithm is forced to explore behind the wall scene in the subsequent iterations, thereby significantly reducing the number of iterations required to detect behind the wall targets.

It is noted that when solving (33) using the modified OMP, the variables $\boldsymbol{\Gamma}, \breve{\mathbf{y}}, \bar{Q}$, and $D$ are equivalent to $\varphi^{(n)} \mathbf{A}, \breve{\mathbf{y}}_{n}, Q$, and $M$, respectively.

\section{Algorithm 1 Modified OMP for $l_{1}$ minimization in TWRI}

\section{Input}

(.) A $\bar{Q} \times D(\bar{Q} \ll D)$ matrix $\boldsymbol{\Gamma}$ which columns are expressed as $\gamma_{j}$

(.) The measurement data vector $\breve{\mathbf{y}}$ of dimension $\bar{Q}$

(.) The sparsity level $P$ of the vector being reconstructed

(.) The wall maximum downrange distance

\section{Procedure}

(1) Initialize the index set $\boldsymbol{\Lambda}_{0}=\left\{\boldsymbol{\lambda}_{\text {wall }}\right\}$, the matrix of chosen atoms $\boldsymbol{X}_{0}=\left\{\boldsymbol{\chi}_{\text {wall }}\right\}$, the vector being reconstructed $\hat{\mathbf{r}}=\mathbf{0}$, and the iteration counter $t=0$.

if $t \neq 0$ then

(2) Find the index $\boldsymbol{\lambda}_{t}$ that solves the following optimization problem,

$$
\boldsymbol{\lambda}_{t}=\arg \max _{j=1, \ldots, \bar{Q}}\left|\left\langle\mathbf{g}_{t-1}, \boldsymbol{\gamma}_{j}\right\rangle\right|
$$

(3) Augment the index set and the matrix of chosen atoms,

$$
\boldsymbol{\Lambda}_{t}=\boldsymbol{\Lambda}_{t-1} \cup\left\{\boldsymbol{\lambda}_{t}\right\} \quad \boldsymbol{X}_{t}=\left[\begin{array}{ll}
\boldsymbol{X}_{t-1} & \boldsymbol{\gamma}_{\boldsymbol{\lambda}_{t}}
\end{array}\right]
$$

\section{end if}

(4) Solve the least square problem to obtain a new signal estimate,

$$
\mathbf{z}_{t}=\arg \min _{\mathbf{z}}\left\|\breve{\mathbf{y}}-\boldsymbol{X}_{t} \mathbf{z}\right\|_{2}^{2}
$$

(5) Calculate the new residual,

$$
\mathbf{g}_{t}=\breve{\mathbf{y}}-\boldsymbol{X}_{t} \mathbf{z}_{t}
$$

(7) Increment $t$, and return to Step 2 if $t<(P-1)$.

(8) The final estimate $\hat{\mathbf{r}}$ is given by,

$$
\hat{\mathbf{r}}\left(\boldsymbol{\Lambda}_{t}\right)=\mathbf{z}_{t}
$$




\section{REFERENCES}

[1] M. G. Amin, Ed., Through-the-Wall Radar Imaging. Boca Raton, FL: CRC Press, 2010.

[2] "Special issue on remote sensing of building interior," IEEE Trans. Geosci. Remote Sens., vol. 47, no. 5, pp. 1270-1420, May 2009.

[3] "Special Issue on Advances in Indoor Radar Imaging," J. Franklin Inst., vol. 345 , no. 6, pp. 556-722, Sep. 2008.

[4] F. Ahmad, M. G. Amin, and P. D. Zemany, "Dual-frequency radars for target localization in urban sensing," IEEE Trans. Aerosp. Electron. Syst., vol. 45, no. 4, pp. 1598-1609, Oct. 2009.

[5] C. Debes, M. G. Amin, and A. M. Zoubir, "Target detection in singleand multiple-view through-the-wall radar imaging," IEEE Trans. Geosci. Remote Sens., vol. 47, no. 5, pp. 1349-1361, May 2009.

[6] S. S. Ram and H. Ling, "Through-wall tracking of human movers using joint Doppler and array processing," IEEE Geosci. Remote Sens. Lett., vol. 5, no. 3, pp. 537-541, Jul. 2008.

[7] Y. Yang and A. E. Fathy, "Development and implementation of a realtime see-through-wall radar system based on FPGA," IEEE Trans. Geosci. Remote Sens., vol. 47, no. 5, pp. 1270-1280, May 2009.

[8] P. C. Chang, R. L. Burkholder, J. L. Volakis, R. J. Marhefka, and Y. Bayram, "High-frequency EM characterization of through-wall building imaging," IEEE Trans. Geosci. Remote Sens., vol. 47, no. 5, pp. 13751387, May 2009.

[9] M. Dehmollaian and K. Sarabandi, "Refocusing through the building walls using synthetic aperture radar," IEEE Trans. Geosci. Remote Sens., vol. 46, no. 6, pp. 1589-1599, Jun. 2008.

[10] F. Soldovieri, G. Prisco, and R. Solimene, "A multi-array tomographic approach for through-wall imaging," IEEE Trans. Geosci. Remote Sens., vol. 46, no. 4, pp. 1192-1199, Apr. 2008.

[11] M. G. Amin and F. Ahmad, "Wideband synthetic aperture beamforming for through-the-wall imaging," IEEE Signal Process. Mag., vol. 25, no. 4, pp. 110-113, Jul. 2008.

[12] C. P. Lai and R. M. Narayanan, "Through-wall imaging and characterization of human activity using ultrawideband (UWB) random noise radar," in Proc. SPIE Symp. Defense Secur., Sensors, C3I Technol. IV Conf., 2005, vol. 5778, pp. 186-195.

[13] F. Ahmad and M. G. Amin, "A noncoherent radar system approach for through-the-wall imaging," in Proc. SPIE Symp. Defense Secur., Sensors, C3I Technol. IV Conf., 2005, vol. 5778, pp. 196-207.

[14] L. P. Song, C. Yu, and Q. H. Liu, "Through-wall imaging (TWI) by radar: 2-D tomographic results and analyses," IEEE Trans. Geosci. Remote Sens., vol. 43, no. 12, pp. 2793-2798, Dec. 2005.

[15] S. Ram, C. Christianson, Y. Kim, and H. Ling, "Simulation and analysis of human micro-Dopplers in through-wall environments," IEEE Trans. Geosci. Remote Sens., vol. 48, no. 4, pp. 2015-2023, Apr. 2010.

[16] H. Stephan, M. Amin, and K. Yemelyanov, "Optimal waveform design for improved indoor target detection in sensing through-the-wall applications," IEEE Trans. Geosci. Remote Sens., vol. 48, no. 7, pp. 2930-2941, Jul. 2010.

[17] A. Sume, M. Gustafsson, M. Herberthson, A. Janis, S. Nilson, J. Rahm, and A. Orborn, "Radar detection of moving targets behind corners," IEEE Trans. Geosci. Remote Sens., vol. 49, no. 6, pp. 2259-2267, Jun. 2011

[18] P. Setlur, M. Amin, and F. Ahmad, "Multipath model and exploitation in through-the-wall and urban radar sensing," IEEE Trans. Geosci. Remote Sens., vol. 49, no. 10, pp. 4021-4034, Oct. 2011.

[19] K. Chetty, G. Smith, and K. Woodbridge, "Through-the-wall sensing of personnel using passive bistatic WiFi radar at standoff distances," IEEE Trans. Geosci. Remote Sens., vol. 50, no. 4, pp. 1218-1226, Apr. 2012.

[20] A. Martone, K. Ranney, and R. Innocenti, "Automatic through the wall detection of moving targets using low-frequency UWB radar," in Proc. IEEE Int. Radar Conf., Washington, DC, May 2010, pp. 39-43.

[21] F. Soldovieri, R. Solimene, and R. Pierri, "A simple strategy to detect changes in through the wall imaging," Progr. Electromagn. Res. M, vol. 7 , pp. 1-13, 2009.

[22] M. G. Amin and F. Ahmad, "Change detection analysis of humans moving behind walls," IEEE Trans. Aerosp. Electron. Syst., to be published.

[23] Y. Yoon and M. G. Amin, "Compressed sensing technique for highresolution radar imaging," in Proc. SPIE, 2008, vol. 6968, pp. 69 681A-169 681A-10.

[24] Q. Huang, L. Qu, B. Wu, and G. Fang, "UWB through-wall imaging based on compressive sensing," IEEE Trans. Geosci. Remote Sens., vol. 48, no. 3, pp. 1408-1415, Mar. 2010.

[25] Y. Yoon and M. G. Amin, "Through-the-wall radar imaging using compressive sensing along temporal frequency domain," in Proc. IEEE ICASSP, Dallas, TX, Mar. 2010, pp. 2806-2809.
[26] M. Leigsnering, C. Debes, and A. Zoubir, "Compressive sensing in through-the-wall radar imaging," in Proc. IEEE ICASSP, Prague, Czech Republic, 2011, pp. 4008-4011.

[27] C. Thajudeen, W. Zhang, and A. Hoorfar, "Time-domain wall parameter estimation and mitigation for through-the-wall radar image enhancement," in Proc. Progr. Electromagn. Res. Symp., Cambridge, MA, Jul. 2010.

[28] Y. Yoon and M. G. Amin, "Spatial filtering for wall-clutter mitigation in through-the-wall radar imaging," IEEE Trans. Geosci. Remote Sens., vol. 47, no. 9, pp. 3192-3208, Sep. 2009.

[29] A. Chandra, D. Gaikwad, D. Singh, and M. Nigam, "An approach to remove the clutter and detect the target for ultra-wideband through-wall imaging," J. Geophys. Eng., vol. 5, no. 4, pp. 412-419, Dec. 2008.

[30] F. Tivive, A. Bouzerdoum, and M. Amin, "An SVD-based approach for mitigating wall reflections in through-the-wall radar imaging," in Proc. IEEE Int. Radar Conf., Kansas City, MO, May 2011, pp. 519-524.

[31] D. Donoho, "Compressed sensing," IEEE Trans. Inf. Theory, vol. 52, no. 4, pp. 1289-1306, Apr. 2006.

[32] E. J. Candes and M. B. Wakin, "An introduction to compressed sampling," IEEE Signal Process. Mag., vol. 25, no. 2, pp. 21-30, Mar. 2008.

[33] E. J. Candes, J. Romberg, and T. Tao, "Robust uncertainty principles: Exact signal reconstruction from highly incomplete frequency information," IEEE Trans. Inf. Theory, vol. 52, no. 2, pp. 489-509, Feb. 2006.

[34] S. Boyd and L. Vandenberghe, Convex Optimization. Cambridge, U.K.: Cambridge Univ. Press, 2004.

[35] E. J. Candes and T. Tao, "Near optimal signal recovery from random projections: Universal encoding strategies," IEEE Trans. Inf. Theory, vol. 52, no. 12 , pp. 5406-5425, Dec. 2006.

[36] S. S. Chen, D. L. Donoho, and M. A. Saunders, "Atomic decomposition by basis pursuit," SIAM J. Sci. Comput., vol. 20, no. 1, pp. 33-61, 1999.

[37] S. Mallat and Z. Zhang, "Matching pursuit with time-frequency dictionaries," IEEE Trans. Signal Process., vol. 41, no. 12, pp. 3397-3415, Dec. 1993.

[38] J. A. Tropp, "Greed is good: Algorithmic results for sparse approximation," IEEE Trans. Inf. Theory, vol. 50, no. 10, pp. 2231-2242, Oct. 2004.

[39] J. A. Tropp and A. C. Gilbert, "Signal recovery from random measurements via orthogonal matching pursuit," IEEE Trans. Inf. Theory, vol. 53 , no. 12, pp. 4655-4666, Dec. 2007.

[40] F. Tivive, M. Amin, and A. Bouzerdoum, "Wall clutter mitigation based on eigen-analysis in through-the-wall radar imaging," in Proc. Int Conf. Digit. Signal Process., Corfu, Greece, Jul. 2011, pp. 1-8.

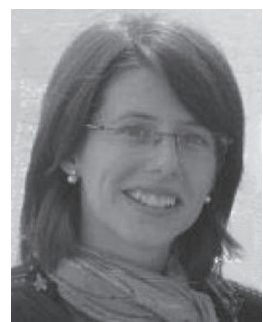

Eva Lagunas (S'09) received the B.S. degree in telecommunications engineering and the M.S. degree from the Technical University of Catalonia (UPC), Barcelona, Spain, in 2009 and 2010, respectively, where she is currently working toward the Ph.D. degree.

She was awarded the Generalitat de Catalunya Grant (FI-DGR 2010) to carry her Ph.D. studies. She is a Research Assistant in the Department of Signal Theory and Communications, UPC, since October 2009. During the summer of 2009, she was a Guest Research Assistant within the Department of Information Engineering, Pisa, Italy. From November 2011 to May 2012, she held a Visiting Researcher Appointment at the Center for Advanced Communications, Villanova University, Villanova, PA. 


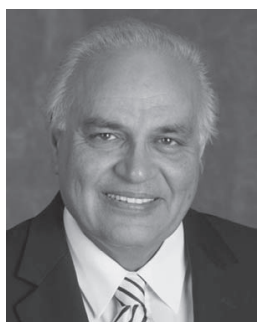

Moeness G. Amin (F'01) received the Ph.D. degree in electrical engineering from University of Colorado, Denver, in 1984.

He has been on the Faculty of the Department of Electrical and Computer Engineering at Villanova University, Villanova, PA, since 1985. In 2002, he became the Director of the Center for Advanced Communications, College of Engineering. He has over 450 journal and conference publications in the areas of wireless communications, time-frequency analysis, smart antennas, waveform design and diversity, interference cancellation in broadband communication platforms, anti-jam GPS, target localization and tracking, direction finding, channel diversity and equalization, ultrasound imaging, and radar signal processing.

Dr. Amin is the Recipient of the 2009 Individual Technical Achievement Award from the European Association of Signal Processing and the Recipient of the 2010 NATO Scientific Achievement Award. He is a Fellow of the International Society of Optical Engineering and a Fellow of the Institute of Engineering and Technology (IET). He is a Recipient of the IEEE Third Millennium Medal, Recipient of the Chief of Naval Research Challenge Award, 2010, Distinguished Lecturer of the IEEE Signal Processing Society, 2003-2004, Active Member of the Franklin Institute Committee on Science and the Arts, Recipient of Villanova University Outstanding Faculty Research Award, 1997, and the Recipient of the IEEE Philadelphia Section Award, 1997. $\mathrm{He}$ is a member of IEEE, SPIE, EURASIP, ION, Eta Kappa Nu, Sigma Xi, and Phi Kappa Phi. He is a Recipient of seven best paper awards. Currently, he serves on the Overview Board of the IEEE TRANSACTIONS ON SignaL PROCESSING. He also serves on the Editorial Board of the EURASIP Signal Processing Journal and the Editorial Board of the Signal Processing Magazine. He was a Plenary Speaker at ICASSP 2010. He was the Special Session CoChair of the 2008 IEEE International Conference on Acoustics, Speech, and Signal Processing. He was the Technical Program Chair of the 2nd IEEE International Symposium on Signal Processing and Information Technology, 2002. He was the General and Organization Chair of the IEEE Workshop on Statistical Signal and Array Processing, 2000. He was the General and Organization Chair of the IEEE International Symposium on Time-Frequency and Time-Scale Analysis, 1994. He was an Associate Editor of the IEEE Transactions on Signal Processing during 1996-1998. He was a member of the IEEE Signal Processing Society Technical Committee on Signal Processing for Communications during 1998-2002. He was a member of the IEEE Signal Processing Society Technical Committee on Statistical Signal and Array Processing during 1995-1997. He was the Guest Editor of the Journal of Franklin Institute September-08 Special Issue on Advances in Indoor Radar Imaging. He was a Guest Editor of the IEEE TRANSACTIONS on Geoscience and Remote Sensing May-09 Special Issue on Remote Sensing of Building Interior, and a Guest Editor of the IET Signal Processing December-09 Special Issue on Time-Frequency Approach to Radar Detection, Imaging, and Classification.

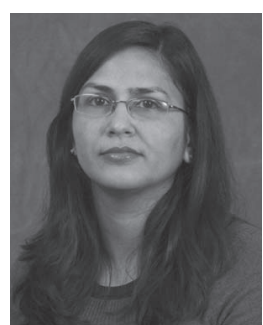

Fauzia Ahmad (S'97-M'97-SM'06) received the M.S. degree in electrical engineering and the Ph.D. degree in electrical engineering from the University of Pennsylvania, Philadelphia, in 1996 and 1997, respectively.

From 1998 to 2000, she was an Assistant Professor in the College of Electrical and Mechanical Engineering, National University of Sciences and Technology, Islamabad, Pakistan. During 2000-2001, she served as an Assistant Professor at Fizaia College of Information Technology, Pakistan. Since 2002, she has been with the Center for Advanced Communications, Villanova University, Villanova, PA, where she is now a Research Associate Professor and the Director of the Radar Imaging Lab. She has over 110 journal and conference publications in the areas of radar imaging, radar signal processing, waveform design and diversity, compressive sensing, array signal processing, sensor networks, ultrasound imaging, and over-the-horizon radar.

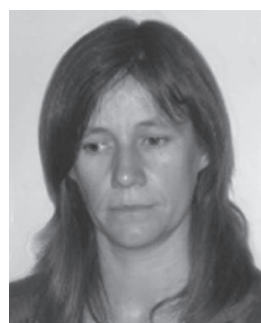

Montserrat Nájar received the electrical engineering and Ph.D. degrees from the Polytechnic University of Catalonia (UPC), Barcelona, Spain, in 1991 and 1996, respectively.

In 1992, she joined the Department of Signal Theory and Communication of the UPC. Since 1997, she has been an Associate Professor at the UPC, where she teaches and coordinates undergraduate and graduate courses in digital communications and signal processing. From 2003 to 2006, she was member of the Board of Directors of the Telecommunications School of Barcelona, ETSETB. Since 2005, she has been a Research Associate in the Centre Tecnològic de Telecomunicació de Catalunya. Her current research interests include signal processing with application to communication systems, array signal processing, and location in wireless systems. She has participated in several IST and Eureka European projects as well as national public and private-funded projects.

Dr. Nájar has been the Guest Editor of the Eurasip Signal Processing journal. She is a Reviewer of the IEEE and the Eurasip Signal Processing journals. 